\begin{tabular}{|c|l|}
\hline Title & Extracting nuclear sizes of medium to heavy nuclei from total reaction cross sections \\
\hline Author(s) & Horiuchi, W.; Hatakey ama, S.; Ebata, S.; Suzuki, Y. \\
\hline Citation & $\begin{array}{l}\text { Physical review C, 93(4), O44611 } \\
\text { https://doi.org/10.1103/PhysRevC.93.044611 }\end{array}$ \\
\hline Issue Date & 2016-04 20 \\
\hline Doc URL & http://hdl.handle.net/2115/62270 \\
\hline Type & article \\
\hline File Information & PhysRevC.93.044611.pdf \\
\hline
\end{tabular}

Instructions for use 


\title{
Extracting nuclear sizes of medium to heavy nuclei from total reaction cross sections
}

\author{
W. Horiuchi, ${ }^{1, *}$ S. Hatakeyama, ${ }^{1}$ S. Ebata, ${ }^{2}$ and Y. Suzuki ${ }^{3,4}$ \\ ${ }^{1}$ Department of Physics, Hokkaido University, Sapporo 060-0810, Japan \\ ${ }^{2}$ Nuclear Reaction Data Centre, Faculty of Science, Hokkaido University, Sapporo 060-0810, Japan \\ ${ }^{3}$ Department of Physics, Niigata University, Niigata 950-2181, Japan \\ ${ }^{4}$ RIKEN Nishina Center, Wako 351-0198, Japan
}

(Received 24 December 2015; published 19 April 2016)

\begin{abstract}
Background: Proton and neutron radii are fundamental quantities of atomic nuclei. To study the sizes of short-lived unstable nuclei, there is a need for an alternative to electron scattering.

Purpose: The recent paper by Horiuchi et al. [Phys. Rev. C 89, 011601(R) (2014)] proposed a possible way of extracting the matter and neutron-skin thickness of light- to medium-mass nuclei using total reaction cross section, $\sigma_{R}$. The analysis is extended to medium to heavy nuclei up to lead isotopes with due attention to Coulomb breakup contributions as well as density distributions improved by paring correlation.

Methods: We formulate a quantitative calculation of $\sigma_{R}$ based on the Glauber model including the Coulomb breakup. To substantiate the treatment of the Coulomb breakup, we also evaluate the Coulomb breakup cross section due to the electric dipole field in a canonical-basis-time-dependent-Hartree-Fock-Bogoliubov theory in the three-dimensional coordinate space.

Results: We analyze $\sigma_{R}$ 's of 103 nuclei with $Z=20,28,40,50,70$, and 82 incident on light targets, ${ }^{1,2} \mathrm{H},{ }^{4} \mathrm{He}$, and ${ }^{12} \mathrm{C}$. Three kinds of Skyrme interactions are tested to generate those wave functions. To discuss possible uncertainty due to the Coulomb breakup, we examine its dependence on the target, the incident energy, and the Skyrme interaction. The proton is a most promising target for extracting the nuclear sizes as the Coulomb excitation can safely be neglected. We find that the so-called reaction radius, $a_{R}=\sqrt{\sigma_{R} / \pi}$, for the proton target is very well approximated by a linear function of two variables, the matter radius and the skin thickness, in which three constants depend only on the incident energy. We quantify the accuracy of $\sigma_{R}$ measurements needed to extract the nuclear sizes.

Conclusions: The proton is the best target because, once the incident energy is set, its $a_{R}$ is very accurately determined by only the matter radius and neutron-skin thickness. If $\sigma_{R}$ 's at different incident energies are measured, one can determine both the proton and neutron radii for unstable nuclei as well. The total reaction cross sections calculated in this paper are given as Supplemental Material for the sake of future measurements.
\end{abstract}

DOI: 10.1103/PhysRevC.93.044611

\section{INTRODUCTION}

Recent systematic studies of the nuclear size provide us with information on not only the saturation property of nuclei but also exotic structure, e.g., deformation, skin, halo, etc. In particular, the nuclear isovector size property, the neutronskin thickness, that is defined by the difference between the neutron and proton radii, $\delta=r_{n}-r_{p}$, has been intensively studied because it is essential for understanding the properties of neutron-rich nuclei and neutron stars, and the equation of state (EOS) of asymmetric nuclear matter [1-7]. However, since the neutron radius is difficult to measure, the neutron-skin thickness is still not as precisely determined as the proton radius [8] that is extracted from the nuclear charge distribution obtained by electron scattering.

One of the most useful observables that reflect the nuclear size is the total reaction $\left(\sigma_{R}\right)$ or interaction cross section $\left(\sigma_{I}\right)$ at high energy. The cross section is accurately measured with recent intense beam facilities and the measurement now reaches beyond the $s d$-shell nuclei [9-11]. Compared to other methods, e.g., proton-nucleus scattering [12-14] and isotope shift measurements [15-17], the method has the great

\footnotetext{
*whoriuchi@nucl.sci.hokudai.ac.jp
}

advantage that it can be applied to almost all nuclei as long as they are produced in sufficient number. Furthermore, a theoretical model to evaluate high energy $\sigma_{R}$ values is well established. The reliability of the reaction model combined with a microscopic structure model has been tested in several examples and has made it possible to reproduce the recent experimental data with no adjustable parameters [18-22].

A charge-changing cross section can be measured by the same setup as that of $\sigma_{I}$. As an alternative to the electron scattering, the charge-changing cross section is expected to provide us with the proton radius of light unstable nuclei [23-25]. However, since the role of a neutron contribution in the chargechanging process, for example, has not yet been understood, the reaction model is not reliable compared to the calculation of $\sigma_{R}$. At present the analysis of the charge-changing cross section requires model- and isotope-dependent corrections to extract the proton radius $[23,25]$.

We here focus on the use of $\sigma_{R}$ to extract the nuclear sizes because both experimental data and theoretical analysis for that cross section appears to be the most reliable. Two of the present authors (W.H. and Y.S.) and Inakura proposed how to determine the matter radius and the neutron-skin thickness using $\sigma_{R}$ [26]. The idea of extracting the neutron-skin thickness is to make use of the different energy-dependences 
of neutron-proton $(n p)$ and proton-proton $(p p)$ total cross sections that are basic ingredients to determine $\sigma_{R}$. A proton target can be a sensitive probe to the neutron distribution because the energy dependence of the $n p$ and $p p$ cross sections is directly reflected in $\sigma_{R}$.

Extending the previous work of Ref. [26] to heavier eveneven nuclei with mass number $A=40-214$, we systematically analyze $\sigma_{R}$ and assess the applicability of the proposed method for extracting the matter radius and neutron-skin thickness of unstable nuclei. In this paper, we examine $\mathrm{Ca}, \mathrm{Ni}, \mathrm{Zr}, \mathrm{Sn}$, $\mathrm{Yb}$, and $\mathrm{Pb}$ isotopes, including both spherical and deformed nuclei. For this purpose we pay due attention to the following two points: One is a Coulomb breakup contribution to $\sigma_{R}$ that may lead to some uncertainty in the extraction of the sizes, and the other is to clarify the sensitivity of the nuclear sizes to $\sigma_{R}$.

The total reaction cross section for a heavier projectile contains the Coulomb breakup contribution that comes from the Coulomb field produced by a target nucleus. In the previous study, the projectile nuclei were limited to light to medium mass nuclei $(8 \leqslant Z \leqslant 28)$, so that the Coulomb interaction between the projectile and the target was ignored. Also, the pairing correlation was ignored in the structure calculation but it may be important in heavier and open-shell nuclei because the level density increases and the mixing of single-particle levels due to the pairing correlation can be important in determining the nuclear shape [27]. By examining both the incident-energy and target dependence of $\sigma_{R}$ with the improved projectile density distribution, we attempt to establish the relationship of $\sigma_{R}$ to the matter radius and neutron-skin thickness, and based on that analysis we propose a possible way of extracting the sizes of medium to heavy unstable nuclei. The study also aims to give data on the nuclear radii and cross sections for the sake of convenience of future measurements.

The paper is organized as follows. Section II explains the reaction and structure models employed in this paper. The inclusion of the Coulomb interaction in the Glauber formalism [28] is discussed in Sec. II A following the treatment of the nuclear breakup process. Since it poses a divergence problem for the Coulomb breakup cross section, a divergence-free approach called the equivalent-photon method (EPM) [29-31] is presented in Sec. II B, which serves as a standard for quantifying the Coulomb breakup cross section in the Glauber formalism. The structure model used in the paper is briefly explained in Sec. II C, where the wave function and the response function of the electric dipole $(E 1)$ field are obtained in the canonical-basis-time-dependent-Hartree-FockBogoliubov (Cb-TDHFB) theory that allows for the pairing correlation [32]. Section III presents results in the following way. We overview a systematic trend of the nuclear sizes of the medium to heavy even-even nuclei in Sec. III A. The Coulomb breakup cross section calculated by the EPM is shown for ${ }^{1,2} \mathrm{H},{ }^{4} \mathrm{He}$, and ${ }^{12} \mathrm{C}$ targets in Sec. III B, and it is compared to the one calculated in the Glauber formalism in Sec. III C. The ratio of the Coulomb breakup cross section to $\sigma_{R}$ is examined in Sec. III D, in order to choose a suitable target that is almost completely free from the Coulomb breakup ambiguity. We examine in Sec. III F the dependence of all of the calculated $\sigma_{R}$ values on the matter radius and the neutron-skin thickness, and discuss in Sec. III G the accuracy required for the measurement of $\sigma_{R}$ in order to extract the neutron-skin thickness of ${ }^{208} \mathrm{~Pb}$. Conclusions are given in Sec. IV.

\section{FORMULATION}

\section{A. Total reaction cross section with Glauber model}

The measurement of $\sigma_{I}$ or $\sigma_{R}$ is mostly performed through high-energy nuclear collisions. The Glauber theory [28] that is based on eikonal and adiabatic approximations gives a simple and good description of high-energy nuclear collision. In the Glauber model, $\sigma_{R}$ is obtained by integrating the reaction probability

$$
P(\boldsymbol{b})=1-\left|e^{i \chi(\boldsymbol{b})}\right|^{2}
$$

over a two-dimensional (2D) impact parameter vector $\boldsymbol{b}$ perpendicular to the beam direction:

$$
\sigma_{R}=\int d \boldsymbol{b} P(\boldsymbol{b})
$$

The optical phase-shift function, $\chi(\boldsymbol{b})$, contains all information on the nucleus-nucleus collision within the Glauber approximation, and its evaluation is vital for the calculation of $\sigma_{R}$.

To incorporate the Coulomb interaction in the Glauber theory, we take the standard approach that directly adds the Coulomb phase to the Glauber nucleon-nucleon $(N N)$ phase-shift function. We start from the usual expression [28] for $\chi(\boldsymbol{b})$ of the projectile and target collision

$$
e^{i \chi(\boldsymbol{b})}=\left\langle\Psi_{0}^{P} \Psi_{0}^{T}\left|\prod_{j \in \mathrm{P}}^{A_{P}} \prod_{k \in \mathrm{T}}^{A_{T}} \mathcal{O}_{N N}\left(\boldsymbol{s}_{j}^{P}-\boldsymbol{s}_{k}^{T}+\boldsymbol{b}\right)\right| \Psi_{0}^{P} \Psi_{0}^{T}\right\rangle,
$$

where $\Psi_{0}^{P}$ and $\Psi_{0}^{T}$ are, respectively, the ground-state wave functions of the projectile and target nucleus with mass number $A_{P}$ and $A_{T}$, and $\mathcal{O}_{N N}$ is related to the $N N$ phase-shift function $\chi_{N N}(\boldsymbol{b})$ as

$$
\mathcal{O}_{N N}(\boldsymbol{b})=e^{i \chi_{N N}(\boldsymbol{b})} \equiv 1-\Gamma_{N N}(\boldsymbol{b}) .
$$

In Eq. (3) $\boldsymbol{s}_{j}^{P}\left(\boldsymbol{s}_{j}^{T}\right)$ denotes a $2 \mathrm{D}$ position vector, perpendicular to the beam direction, of the $j$ th nucleon of the projectile (target) from its center of mass. The profile function $\Gamma_{N N}$ describes the $N N$ collision at incident energy $E$, and it is usually parametrized as [33]

$$
\Gamma_{N N}(\boldsymbol{b})=\frac{1-i \alpha_{N N}}{4 \pi \beta_{N N}} \sigma_{N N}^{\text {tot }} \exp \left[-\frac{\boldsymbol{b}^{2}}{2 \beta_{N N}}\right],
$$

where $\alpha_{N N}$ is the ratio of the real to the imaginary part of the $N N$ scattering amplitude of forward angle, $\beta_{N N}$ is the slope parameter of the $N N$ elastic scattering differential cross section, and $\sigma_{N N}^{\text {tot }}$ is the total cross section of the $N N$ scattering. Note that the $n p$ profile function is different from the $p p$ one. The $n n$ profile function is taken the same as that for $p p$. These parameters are tabulated in Ref. [34] for a wide energy range.

By including the Coulomb interaction, $\mathcal{O}_{N N}\left(\boldsymbol{s}_{j}^{P}-\boldsymbol{s}_{k}^{T}+\boldsymbol{b}\right)$ of Eq. (3) is modified to

$$
\exp \left\{i\left[\chi_{N N}\left(\boldsymbol{s}_{j}^{P}-\boldsymbol{s}_{k}^{T}+\boldsymbol{b}\right)+\epsilon_{j} \epsilon_{k} \Delta \chi_{C}\left(\boldsymbol{s}_{j}^{P}-\boldsymbol{s}_{k}^{T}+\boldsymbol{b}\right)\right]\right\},
$$

where $\epsilon_{j} \epsilon_{k}$ is 1 when both $j$ and $k$ stand for protons but otherwise 0 , and $\Delta \chi_{C}$ is the Coulomb phase causing the 
breakup

$$
\Delta \chi_{C}(\boldsymbol{s}+\boldsymbol{b})=2 \eta \ln \left(\frac{|\boldsymbol{s}+\boldsymbol{b}|}{b}\right),
$$

where $\eta=e^{2} / \hbar v$ is the Sommerfeld parameter with $v$ being the projectile's velocity. Defining $\Gamma_{C}=1-e^{i \Delta \chi_{C}}$, we have

$$
\Gamma_{C}(\boldsymbol{s}+\boldsymbol{b})=1-\left(\frac{|\boldsymbol{s}+\boldsymbol{b}|}{b}\right)^{2 i \eta},
$$

and may write the total optical phase-shift function as

$$
e^{i \chi(\boldsymbol{b})}=\left\langle\Psi_{0}^{P} \Psi_{0}^{T}\left|\prod_{j \in P}^{A_{P}} \prod_{k \in T}^{A_{T}}\left[1-\Gamma_{\mathrm{tot}}\left(\boldsymbol{s}_{j}^{P}-\boldsymbol{s}_{k}^{T}+\boldsymbol{b}\right)\right]\right| \Psi_{0}^{P} \Psi_{0}^{T}\right\rangle
$$

with

$$
\begin{aligned}
1-\Gamma_{\text {tot }}\left(s_{j}^{P}-s_{k}^{T}+b\right)= & {\left[1-\Gamma_{N N}\left(s_{j}^{P}-s_{k}^{T}+b\right)\right] } \\
& \times\left[1-\epsilon_{j} \epsilon_{k} \Gamma_{C}\left(s_{j}^{P}-s_{k}^{T}+b\right)\right] .
\end{aligned}
$$

An evaluation of $\chi(\boldsymbol{b})$ through Eq. (9) is in general not easy as it involves multiple integration of a many-variable function. The integration can be performed with a Monte Carlo technique as shown in Ref. [35]. For an extensive study, however, we use an optical-limit approximation (OLA) that takes account of the leading order of the cumulant expansion [28]

$$
e^{i \chi(\boldsymbol{b})} \sim \exp \left(i \chi^{(N)}(\boldsymbol{b})+i \chi^{(C)}(\boldsymbol{b})+i \chi^{(N C)}(\boldsymbol{b})\right),
$$

where the nuclear $(N)$, Coulomb $(C)$, and nuclear-Coulomb interference $(N C)$ phases are defined by

$$
\begin{aligned}
i \chi^{(N)}(\boldsymbol{b})= & -\iint d \boldsymbol{r}^{P} d \boldsymbol{r}^{T} \rho^{P}\left(\boldsymbol{r}^{P}\right) \rho^{T}\left(\boldsymbol{r}^{T}\right) \\
& \times \Gamma_{N N}\left(\boldsymbol{s}^{P}-\boldsymbol{s}^{T}+\boldsymbol{b}\right), \\
i \chi^{(C)}(\boldsymbol{b})= & -\iint d \boldsymbol{r}^{P} d \boldsymbol{r}^{T} \rho_{p}^{P}\left(\boldsymbol{r}^{P}\right) \rho_{p}^{T}\left(\boldsymbol{r}^{P}\right) \\
& \times \Gamma_{C}\left(\boldsymbol{s}^{P}-\boldsymbol{s}^{T}+\boldsymbol{b}\right), \\
i \chi^{(N C)}(\boldsymbol{b})= & \iint d \boldsymbol{r}^{P} d \boldsymbol{r}^{T} \rho_{p}^{P}\left(\boldsymbol{r}^{P}\right) \rho_{p}^{T}\left(\boldsymbol{r}^{T}\right) \\
& \times \Gamma_{N N}\left(\boldsymbol{s}^{P}-\boldsymbol{s}^{T}+\boldsymbol{b}\right) \Gamma_{C}\left(\boldsymbol{s}^{P}-\boldsymbol{s}^{T}+\boldsymbol{b}\right) .
\end{aligned}
$$

The integration is carried out using the density distributions of the projectile and target, $\rho^{P}\left(\boldsymbol{r}^{P}\right)$ and $\rho^{T}\left(\boldsymbol{r}^{T}\right)$, and the point-proton densities of the projectile and target, $\rho_{p}^{P}\left(\boldsymbol{r}^{P}\right)$ and $\rho_{p}^{T}\left(\boldsymbol{r}^{T}\right)$. It should be noted that the expression includes the Coulomb breakup effect of not only the projectile but also the target.

For a later analysis, it is useful to decompose the reaction probability into three terms:

$$
P(\boldsymbol{b})=P^{(N)}(\boldsymbol{b})+P^{(C)}(\boldsymbol{b})+P^{(N C)}(\boldsymbol{b})
$$

with

$$
P^{(N)}(\boldsymbol{b})=\left(1-\left|e^{i \chi^{(N)}(\boldsymbol{b})}\right|^{2}\right)
$$

$$
\begin{aligned}
P^{(C)}(\boldsymbol{b}) & =\left|e^{i \chi^{(N)}(\boldsymbol{b})}\right|^{2}\left(1-\left|e^{i \chi^{(C)}(\boldsymbol{b})}\right|^{2}\right), \\
P^{(N C)}(\boldsymbol{b}) & =\left|e^{i \chi^{(N)}(\boldsymbol{b})+i \chi^{(C)}(\boldsymbol{b})}\right|^{2}\left(1-\left|e^{i \chi^{(N C)}(\boldsymbol{b})}\right|^{2}\right) .
\end{aligned}
$$

Though this decomposition may not be unique, it is physically reasonable because the interference term given by Eq. (18) is very small as shown in Ref. [36]. Then $\sigma_{R}$ given in the leading-order approximation reads

$$
\sigma_{R}=\sigma_{R}^{(N)}+\sigma_{R}^{(C)}+\sigma_{R}^{(N C)},
$$

where

$$
\sigma_{R}^{(X)}=\int d \boldsymbol{b} P^{(X)}(\boldsymbol{b})
$$

with $X=N, C$, or $N C$. If the nuclear interaction is turned off, $\sigma_{R}$ turns out to be

$$
\sigma_{C}=\int d \boldsymbol{b}\left(1-\left|e^{i \chi^{(C)}(\boldsymbol{b})}\right|^{2}\right),
$$

which is nothing but the Coulomb breakup cross section in the OLA of the Glauber theory.

In this paper we consider the targets, ${ }^{1,2} \mathrm{H},{ }^{4} \mathrm{He}$, and ${ }^{12} \mathrm{C}$. We use the ${ }^{12} \mathrm{C}$ density distribution obtained in Ref. [37], and the density distributions of ${ }^{2} \mathrm{H}$, and ${ }^{4} \mathrm{He}$ obtained by few-body calculations with the Argonne V8' potential plus a central three-body force [38]. We fit these densities by several Gaussian functions, and tabulate the parameters in Supplemental Material [39]. Since the OLA works well for proton-nucleus scattering in which the $N N$ multiple-scattering effect can be neglected [35], we employ the OLA for the ${ }^{1} \mathrm{H}$ (proton) target. For the other targets we employ the nucleon-target formalism in the Glauber theory (NTG) $[36,40]$, to describe the nuclear breakup part, which is formulated based on the nucleon-target $(N T)$ interaction, $\Gamma_{N T}$, representing the interaction between the nucleon and the target. The NTG approximation requires the same inputs as those used in the ordinary OLA of Eq. (12) and reproduces $\sigma_{R}$ better than the OLA that misses some higher-order terms of $\Gamma_{N N}$. For example, $\sigma_{R}$ of ${ }^{12} \mathrm{C}+{ }^{12} \mathrm{C}$ calculated with the NTG are very much improved in a wide energy range [37].

\section{B. Equivalent-photon method}

In Sec. II A, we give the formulation to take into account the Coulomb breakup process in the Glauber model. However, $P^{(C)}(\boldsymbol{b})$ leads to a logarithmic divergence if the impact parameter integration is performed to infinity with use of the eikonal Coulomb phase [36], which is due to the neglect of the energy conservation inherent in the adiabatic approximation. A nonadiabatic treatment of the Coulomb breakup processes is needed to correctly describe the effect of the long-range Coulomb force. Though several attempts to avoid the divergence are performed in a few-body system [41-45], they are too computer-time expensive to apply to a nucleus-nucleus case. A simple, practical way to avoid the divergence problem is to replace the Coulomb phase that leads to the divergence by a divergence-free perturbative term assuming the dominance of the $E 1$ field. This idea called the Coulomb-corrected eikonal approximation works well and still makes it possible to employ the eikonal approximation [46-48]. However, it is 
only applicable to some special cases, for example, two- or three-body models for a halo nucleus [48-50], and it will not give us a convenient formula for a more general case because the knowledge of the photoabsorption cross section of each many-body final state is required.

A systematic trend of the Coulomb breakup cross section can be discussed by using the EPM [29-31], which gives the simplest estimate of the Coulomb breakup contribution if the photoabsorption response of the projectile nucleus is available. The method is widely used and succeeds in explaining the large Coulomb breakup cross sections of halo nuclei [51]. In fact it is used to extract the $E 1$ strength function of a halo nucleus from the measurement of the neutron removal cross section through the Coulomb breakup process [52,53]. In the EPM the reaction probability for the Coulomb excitation, $P_{C}(\boldsymbol{b})$, is expressed with the photoabsorption cross section, $\sigma_{\gamma}(\omega)$, multiplied by the number of photons per unit area per unit frequency, $N(\boldsymbol{b}, \omega)$, as

$$
P_{C}(\boldsymbol{b})=\int_{0}^{\infty} d \omega \sigma_{\gamma}(\omega) N(\boldsymbol{b}, \omega) .
$$

If the photoabsorption is dominated by the $E 1$ response, $\sigma_{\gamma}(\omega)$ is given by the $E 1$ strength function, $d B(E 1) / d E$, as [27]

$$
\sigma_{\gamma}(\omega)=\frac{16 \pi^{3} \omega}{9} \frac{d B(E 1)}{d E}
$$

and $N(\boldsymbol{b}, \omega)$ is given by

$$
N(\boldsymbol{b}, \omega)=\frac{Z_{T}^{2} e^{2}}{\pi^{2} \hbar c}\left(\frac{c}{v}\right)^{2} \frac{\xi^{2}}{\omega b^{2}}\left[K_{1}^{2}(\xi)+\frac{1}{\gamma^{2}} K_{0}^{2}(\xi)\right]
$$

with the Lorentz factor $\gamma$ and $\xi=b \omega / \gamma v$, where $K_{0}$ and $K_{1}$ are the modified Bessel functions of zeroth and first orders, respectively. In general, as the incident energy decreases, we have to take into account Coulomb multipole breakup contributions other than the E1 multipole [29]. Evaluating the contributions of all the multipoles is beyond the scope of this paper. Note that $P_{C}(\boldsymbol{b})$ and $P^{(C)}(\boldsymbol{b})$ of Eq. (17) show quite different behavior, especially at low incident energy.

The EPM is formulated in a semiclassical way and $P_{C}(\boldsymbol{b})$ exceeds unity at small $b$. The Coulomb excitation of a giant resonance was treated in a perturbation theory using the eikonal approximation [54]. This theory is more satisfactory in that the suppression of $P_{C}(\boldsymbol{b})$ at small $b$ is accounted for by the absorption of the imaginary part of the optical potential between the nuclei. The corresponding suppression is represented by the factor $\left|e^{i \chi^{(N)}(\boldsymbol{b})}\right|^{2}$ of Eq. (17) in our formulation. Since our main concern here is the long-range behavior of $P^{(C)}(\boldsymbol{b})$ leading to the divergence, we do not pay much attention to the detail of the short-range behavior of the Coulomb breakup probability and simply introduce a sharp cutoff, $b_{\min }$, for the sake of simplicity. The Coulomb breakup cross section in the EPM is then defined as

$$
\sigma_{C}=\int_{|\boldsymbol{b}| \geqslant b_{\min }} d \boldsymbol{b} P_{C}(\boldsymbol{b}),
$$

where $b_{\min }$ is taken as $\sqrt{5 / 3}\left(r_{m}^{P}+r_{m}^{T}\right)$ using $r_{m}^{P}\left(r_{m}^{T}\right)$, the root-mean-square (rms) radius of the projectile (target). Since the contribution of the Coulomb interference term to $\sigma_{R}$ is small [36,55], the calculated Coulomb breakup cross section is added incoherently to the cross section $\sigma_{R}^{(N)}$ obtained only with the nuclear interaction.

\section{Densities and $E 1$ response in Cb-TDHFB theory}

Nuclear pairing correlations are important to account for the odd-even mass difference as well as the separation energy, especially for heavy nuclei [56]. The TDHFB theory is powerful to describe the dynamics of a nuclear system including pairing. However, due to its huge numerical cost as well as the complexity of numerical calculations, its application is limited to only special cases. The Cb-TDHFB was developed to study the ground-state property as well as nuclear response for a wide nuclear mass range [32]. The efficiency of this method is confirmed by, e.g., the $E 1$ response function of ${ }^{172} \mathrm{Yb}$, for which the $\mathrm{Cb}$-TDHFB gives results consistent with the quasi-particle random phase approximation (QRPA) [57]. The numerical cost of the Cb-TDHFB is found to be about $1 / 1000$ of that of QRPA [58]. We briefly describe how we obtain the density distribution and the $E 1$ response function that are needed for calculating $\sigma_{R}$. See Refs. [32,59] for details.

For a description of the ground state, we adopt a Skyrme energy density functional with three parameter sets of SkM* [60], SLy4 [61], and SkI3 [62]. The SkM* is known to account well for the properties of deformed nuclei because its parameter is determined to reproduce the fission barrier of ${ }^{240} \mathrm{Pu}$. The SLy4 is superior to $\mathrm{SkM}^{*}$ in reproducing the properties of neutron-rich nuclei. In addition to these, we employ the SkI3 parameter set, which simulates the single-particle levels of the relativistic mean-field model, giving different characteristics especially in the $\mathrm{Pb}$ region.

Each single-particle wave function is represented by the canonical state that diagonalizes the density matrix [32]. To describe nuclear deformations the three-dimensional (3D) Cartesian coordinate space is chosen, which is discretized in square mesh of $d$ in a sphere with radius of $R$. Thus, each canonical state is represented by three discrete indexes $\left(i_{x}, i_{y}, i_{z}\right)$ for the 3D mesh points; $(x, y, z)=\left(i_{x}, i_{y}, i_{z}\right) \times d$. In the present work, we choose $d=1 \mathrm{fm}$ and $R=15 \mathrm{fm}$ for all isotopes. For the ground state, the self-consistent solution is obtained by the procedure written in Sec. VC of Ref. [32]. The intrinsic one-body density is expressed by the single-particle wave functions. For the Glauber model calculation, we need the one-body density distributions in the laboratory frame. As was done in Ref. [21], the angle-averaged intrinsic one-body densities are used as the projectile density distributions.

The $E 1$ strength function is obtained by applying a weak impulse external field to the initial state, and then its timeevolution is described by the Cb-TDHFB equation [32]. The linear response is calculated by the prescription given in Ref. [63].

\section{RESULTS}

\section{A. Matter radii and neutron-skin thickness}

We take up 103 even-even nuclei for each of the Skyrme interactions, ${ }^{40-60} \mathrm{Ca},{ }^{56-84} \mathrm{Ni},{ }^{80-122} \mathrm{Zr},{ }^{100-140} \mathrm{Sn},{ }^{156-196} \mathrm{Yb}$, and ${ }^{190-214} \mathrm{~Pb}$, covering mainly the neutron-rich region. To avoid 


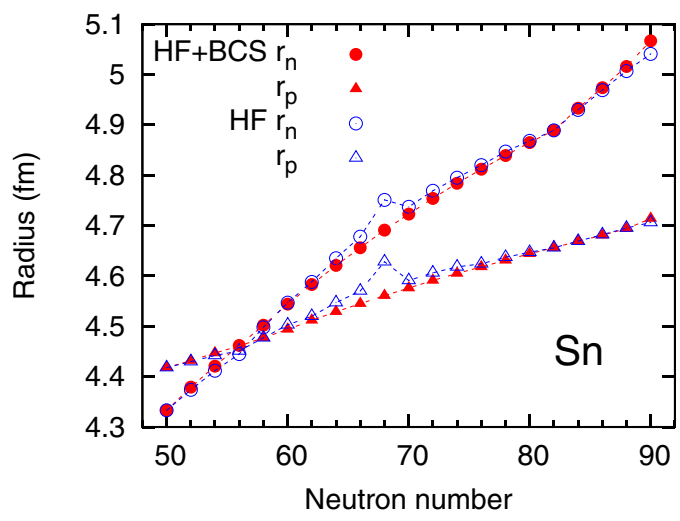

FIG. 1. Neutron and proton rms radii of $\mathrm{Sn}$ isotopes calculated with $\mathrm{HF}+\mathrm{BCS}$ and $\mathrm{HF}$ theory. The SkM* interaction is used.

spurious continuum couplings in the BCS model $[64,65]$, we consider only those nuclei that give the Fermi-energy higher than $2 \mathrm{MeV}$. To know the effect of the pairing correlation on the nuclear sizes, we compare in Fig. 1 the neutron and proton rms radii, $r_{n}$ and $r_{p}$, of $\mathrm{Sn}$ isotopes calculated by $\mathrm{HF}$ and $\mathrm{HF}+\mathrm{BCS}$ methods. The radii calculated with or without the pairing correlation show almost identical results except for $N=68$. This difference is due to the deformation that enhances the matter radius $[20,21]$. The deformation of the HF ground state reaches a maximum at $N=68$ (the quadrupole deformation parameter, $\beta_{p}=0.281$ and $\beta_{n}=0.279$ for proton and neutron, respectively), whereas the $\mathrm{HF}+\mathrm{BCS}$ predicts no deformation. Since the proton and neutron deformation parameters of ${ }^{118} \mathrm{Sn}$ are almost the same in the HF calculation, no neutron-skin thickness develops at $N=68$.

Let us define the matter radius, $r_{m}(N, Z)$, and the neutronskin thickness, $\delta(N, Z)$, using the proton and neutron rms radii, $r_{p}(N, Z)$ and $r_{n}(N, Z)$, by

$$
\begin{aligned}
r_{m}(N, Z) & =\sqrt{\frac{Z}{A} r_{p}^{2}(N, Z)+\frac{N}{A} r_{n}^{2}(N, Z)}, \\
\delta(N, Z) & =r_{n}(N, Z)-r_{p}(N, Z) .
\end{aligned}
$$

Figures 2 and 3 display $r_{m}(N, Z)$ and $\delta(N, Z)$ calculated with SkM*, SLy4, and SkI3 interactions. The $r_{m}(N, Z)$ value roughly follows an $(N+Z)^{1 / 3}$ line for all the cases as expected. The $\delta(N, Z)$ value shows some interaction dependence. It grows as the asymmetry parameter, $(N-Z) /(N+Z)$, increases. A spread of $\delta(N, Z)$ becomes larger as the asymmetry parameter approaches that of the region far from the stability. Since the nuclear sizes are interaction-dependent, it is not easy to find out how $r_{m}$ and $\delta$ depend on $Z$ and $N$.

\section{B. Coulomb breakup cross sections by EPM}

First we discuss a systematic trend of the Coulomb breakup cross sections using the EPM, Eq. (25). Figure 4 plots the Coulomb breakup cross section by a proton target. The incident energies are 200 and $1000 \mathrm{MeV}$. Here the incident energy means the energy per nucleon and is simply written in $\mathrm{MeV}$ throughout this paper. The magnitude of $\sigma_{C}$ is found to be very small, at most $2 \mathrm{mb}$, which will be about $0.1 \%$ of $\sigma_{R}$. The cross section increases gradually as $N$ increases, following the enhancement of $r_{m}$ shown in Fig. 2. Most of the E1 strength come from the giant resonance region. We observe a sudden increase of the cross section for $\mathrm{Ni}$ isotopes beyond $N=50$ and $\mathrm{Sn}$ isotopes beyond $N=82$, even larger in the case of the low-incident energy, especially when the SkI3 interaction is used. This is due to the enhancement of the low-lying $E 1$ strength, as discussed in Ref. [59]. The photon number (24) concentrates at the low-excitation energy and decreases rapidly with increasing excitation energy [29]. In that case, the cross section is more sensitive to the low-energy strength rather than the strength in the giant resonance region. In the high-incident energy, the photon number decreases more slowly than in the low-incident energy. Though the Coulomb breakup cross section by protons is small, it shows some dependence on the incident energy and also on the interaction used.

The upper panels of Fig. 5 display the Coulomb breakup cross sections on ${ }^{2} \mathrm{H},{ }^{4} \mathrm{He}$, and ${ }^{12} \mathrm{C}$ targets at $1000 \mathrm{MeV}$ incident energy. The cross section behaves as a function of $N$ similarly to the case of the proton target but its magnitude is approximately scaled by $Z_{T}^{2}$, as noted in Eq. (24). In the Coulomb breakup of the nucleus-nucleus collision, the target nucleus can also be excited by the Coulomb force from the projectile. That cross section contributes to $\sigma_{R}$ and it is readily

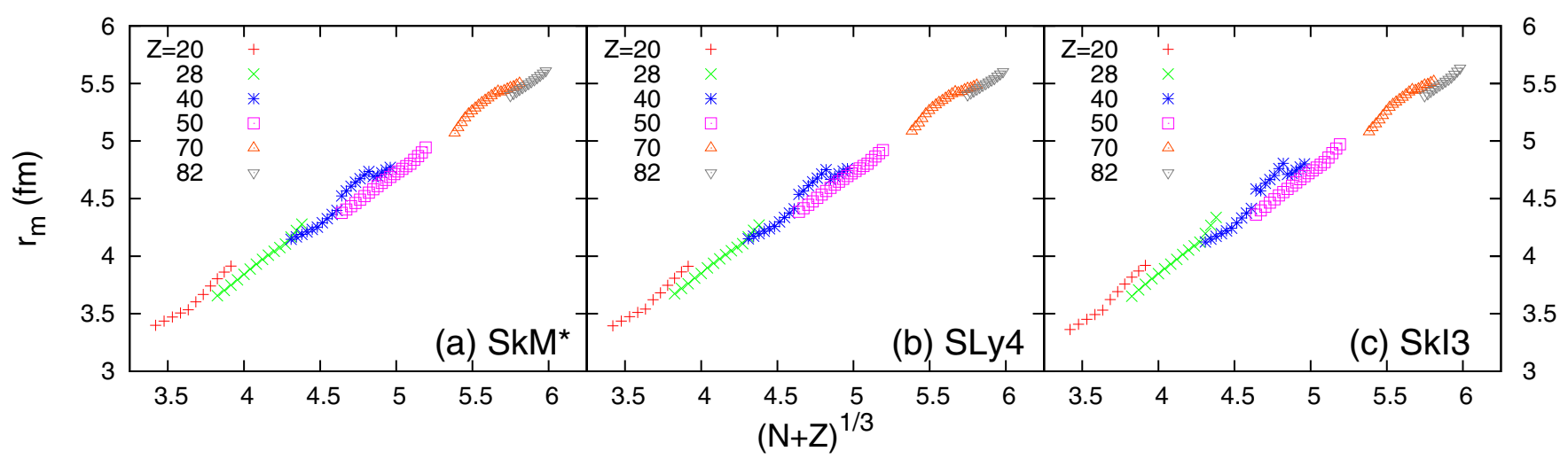

FIG. 2. Matter radius of even-even nucleus as a function of $(N+Z)^{1 / 3}$. Three Skyrme parameter sets are used: (a) SkM*, (b) SLy4, and (c) SkI3. 


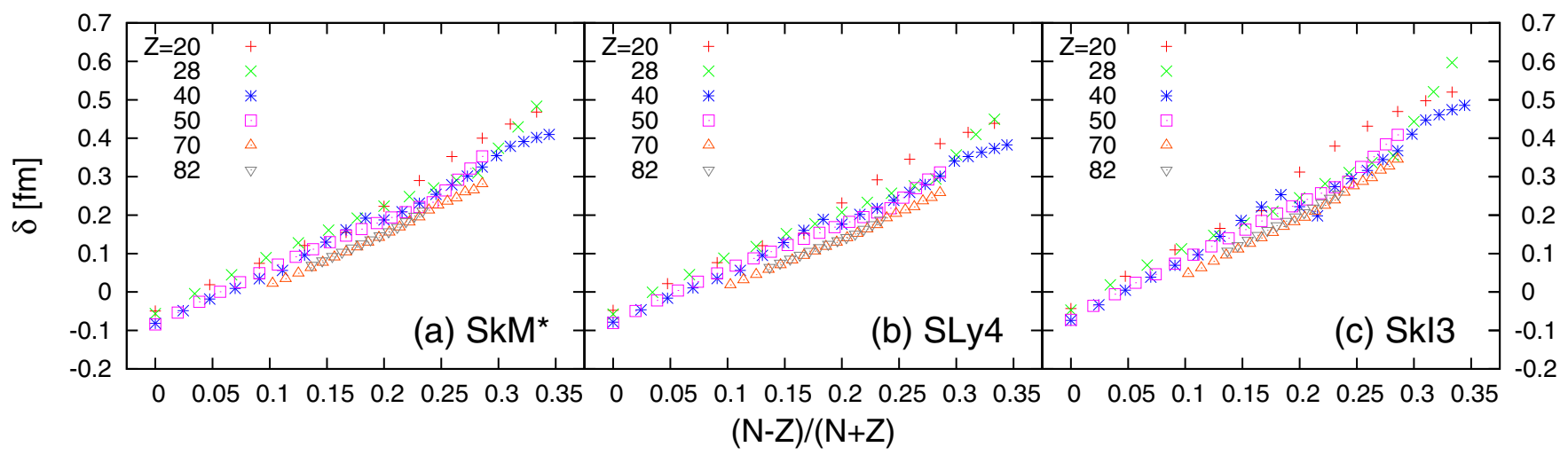

FIG. 3. Neutron-skin thickness of even-even nucleus as a functions of $(N-Z) /(N+Z)$. Three Skyrme parameter sets are used: (a) SkM*, (b) SLy4, and (c) SkI3.

calculated by exchanging the role of the target and projectile in Eqs. (22) and (24), once the photoabsorption cross section or $E 1$ strength function of the target nucleus is available. We make use of the following sources: the cross section of Ref. [66] for ${ }^{2} \mathrm{H}$, the $a b$ initio calculation [21] for ${ }^{4} \mathrm{He}$, and the $E 1$ strength function calculated by the Cb-TDHFB for ${ }^{12} \mathrm{C}$. The lower panels of Fig. 5 exhibit the Coulomb breakup cross sections of these targets as a functions of $N$ of the projectile. Since the cross section depends on $Z_{P}^{2}$ and $Z_{P}$ considered here is large, the Coulomb breakup contribution of the target is much larger than that of the projectile, especially when ${ }^{2} \mathrm{H}$ is a target. This is in exactly the same situation as the Coulomb breakup of a halo nucleus by a heavy target because ${ }^{2} \mathrm{H}$ is a weakly bound nucleus. Hereafter we let the Coulomb breakup cross section by EPM indicate a sum of both contributions, the projectile breakup and the target breakup.

\section{Coulomb breakup-Glauber model versus EPM}

It is important for our purpose to establish a sound procedure for taking account of the Coulomb breakup process in the Glauber model. We here compare the results with the Glauber model and the EPM. To avoid the divergence that the Glauber Coulomb eikonal phase causes, we introduce a cutoff impact parameter $b_{\max }$ such that $\sigma_{R}^{(C)}$ of the Glauber model

$$
\sigma_{R}^{(C)}=\int_{|\boldsymbol{b}| \leqslant b_{\max }} d \boldsymbol{b} P^{(C)}(\boldsymbol{b})
$$

reproduces $\sigma_{C}$ obtained by the EPM. Note that $P^{(C)}(\boldsymbol{b})$ becomes vanishingly small for $b$ where the projectile and the target overlap significantly because the so-called survival probability [67,68], $\left|e^{i \chi^{(N)}(b)}\right|^{2}$, becomes so small. See Eq. (17).

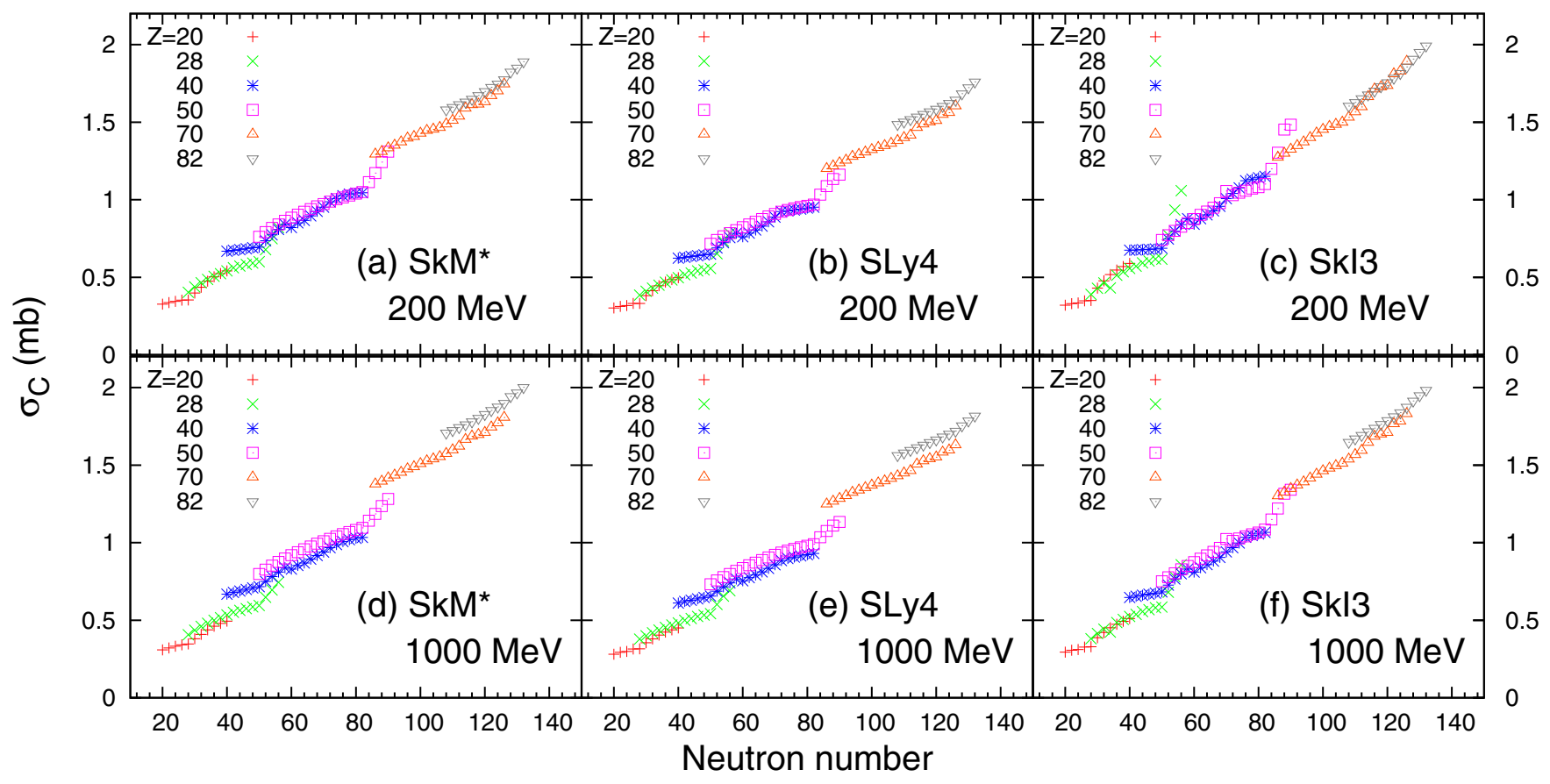

FIG. 4. Coulomb breakup cross sections by EPM for projectile nucleus incident on proton at (top) 200 and (bottom) $1000 \mathrm{MeV}$. The projectile density is obtained with three Skyrme interactions: (Left) SkM*, (Center) SLy4, and (Right) SkI3. 

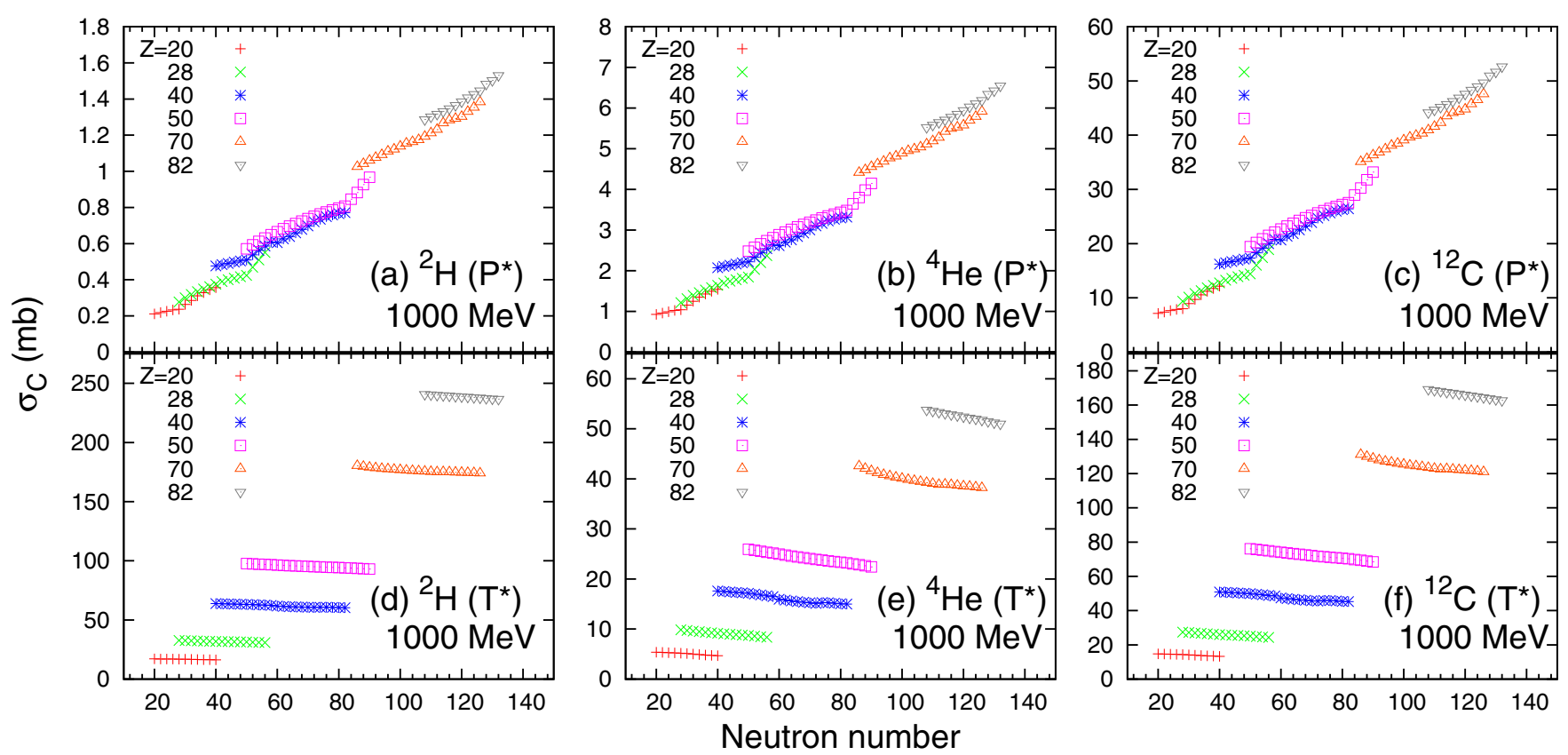

FIG. 5. EPM calculation of Coulomb breakup cross sections for (upper) projectile by target and for (lower) target by projectile at $1000 \mathrm{MeV}$ incident energy. Three different targets are employed; (Left) ${ }^{2} \mathrm{H}$, (Center) ${ }^{4} \mathrm{He}$, and (Right) ${ }^{12} \mathrm{C}$. The projectile density is obtained with the $\mathrm{SkM}^{*}$ interaction.

In order to see the contribution of the Coulomb breakup to the total reaction cross section, we plot in Fig. 6 the decomposition of the total reaction probability into the nuclear and Coulomb breakup probabilities in the case of ${ }^{208} \mathrm{~Pb}+{ }^{12} \mathrm{C}$ collision at $1000 \mathrm{MeV}$. The decomposition is made using Eqs. (16)-(18). The contribution of the interference term is so small that it is not shown. The Coulomb breakup contribution to the cross section arises from the nuclear surface where $P^{(N)}$ falls off but its magnitude is very small compared to the nuclear one.

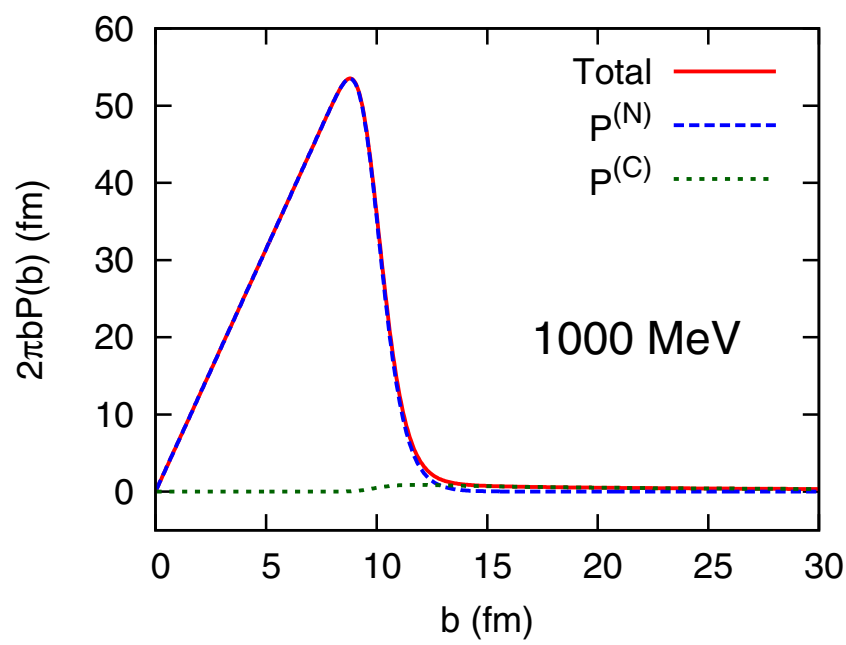

FIG. 6. Glauber model calculation of the total reaction probability and its decomposition to the nuclear and Coulomb breakup probabilities for ${ }^{208} \mathrm{~Pb}+{ }^{12} \mathrm{C}$ collision at $1000 \mathrm{MeV}$ incident energy. The SkM* interaction is used.
For a more quantitative discussion on the Coulomb breakup process, we compare in Fig. 7 the ${ }^{208} \mathrm{~Pb}+{ }^{12} \mathrm{C}$ Coulomb breakup

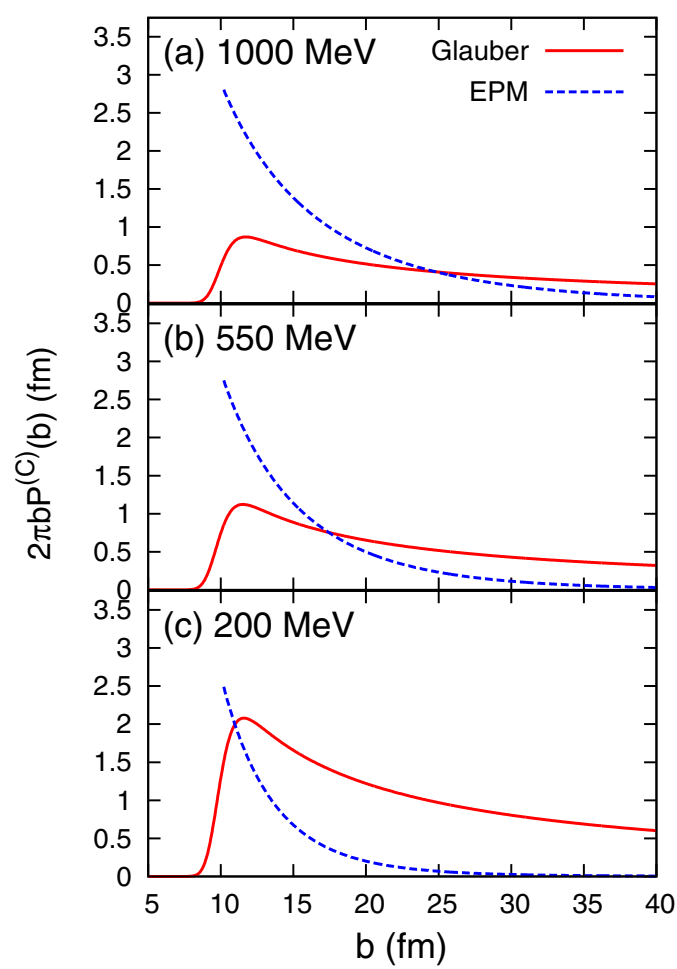

FIG. 7. Comparison of ${ }^{208} \mathrm{~Pb}+{ }^{12} \mathrm{C}$ Coulomb breakup probabilities calculated by the Glauber and EPM models at three incident energies (a) 1000, (b) 550, and (c) $200 \mathrm{MeV}$. The cutoff parameter of $\mathrm{EPM}$ is $b_{\min }=10.2 \mathrm{fm}$. The $\mathrm{SkM}^{*}$ interaction is used. 
probabilities calculated by the Glauber model and EPM at three incident energies of 1000, 550, and $200 \mathrm{MeV}$. The probabilities calculated by the Glauber model rise from a little inside of $b_{\text {min }}=10.2 \mathrm{fm}$ and decrease asymptotically as $1 / b$ [36]. At the high-incident energy, the Coulomb breakup probability given by the Glauber model shows behavior similar to that of EPM. This is understood by considering the spectrum of $N(\boldsymbol{b}, \omega)$. When the projectile's velocity is high and $\eta$ is small, the photon number decreases rapidly with increasing multipolarity [29]. As the incident energy decreases below $200 \mathrm{MeV}$, the $b$ dependence of the Coulomb breakup probability is quite different from that of the EPM. This can be seen by expanding the Coulomb breakup profile function (8) at sufficiently large $b[36]$

$$
\begin{aligned}
\Gamma_{C}(\boldsymbol{s}+\boldsymbol{b}) \rightarrow & -\frac{2 i \eta}{b}(\boldsymbol{s} \cdot \hat{\boldsymbol{b}})-\frac{i \eta}{b^{2}}\left\{s^{2}-2(\boldsymbol{s} \cdot \hat{\boldsymbol{b}})^{2}\right\} \\
& +\frac{2 \eta^{2}}{b^{2}}(\boldsymbol{s} \cdot \hat{\boldsymbol{b}})^{2}+\cdots
\end{aligned}
$$

where $\hat{\boldsymbol{b}}=\boldsymbol{b} / \boldsymbol{b}$. The first term does not contribute to the cross section. For small $\eta$, the second term that induces the dipole excitation dominantly contribute to the Coulomb breakup probability. For large $\eta$, however, the other terms cannot be neglected, giving a contribution of other multipoles to the Coulomb breakup. As the incident energy decreases, the $\eta$ value increases, and thereby the $E 1$ contribution becomes relatively smaller and other multipole effects are amplified, consistently with Ref. [29].

As seen above, the E1 approximation with the EPM reasonably well takes account of the Coulomb breakup process when the incident energy is high enough, say above $500 \mathrm{MeV}$. At the lower incident energy, the other multipole effects $(E 2, E 3, \ldots)$ should be considered for a quantitative evaluation of the Coulomb breakup cross section. Even though we could calculate response functions of all multipoles, the resulting Coulomb breakup cross section would not be able to be free from some uncertainty due to, e.g., the interaction to be used in that calculation.

The Coulomb breakup probability calculated in Ref. [36] employs a point charge assumption for the target. The Coulomb breakup probability is then much larger than that of the present calculation and can significantly contribute to the total reaction probability. The reason for this is apparently because the Sommerfeld parameter becomes quite large under the point charge assumption, and other multipoles higher than $E 1$ contribute significantly to the reaction probability as noted above.

\section{Contribution of Coulomb breakup to total reaction cross section}

As discussed in Secs. III B and III C, neither the EPM nor Glauber model gives a complete procedure to quantitatively evaluate the contribution of the Coulomb breakup to $\sigma_{R}$ for arbitrary incident energies. The EPM has no problem in so far as the $E 1$ response predominantly contributes to the Coulomb breakup. However, other multipoles contribute as the incident energy decreases and calculating those responses for each nuclear system is not very practical. The Glauber model

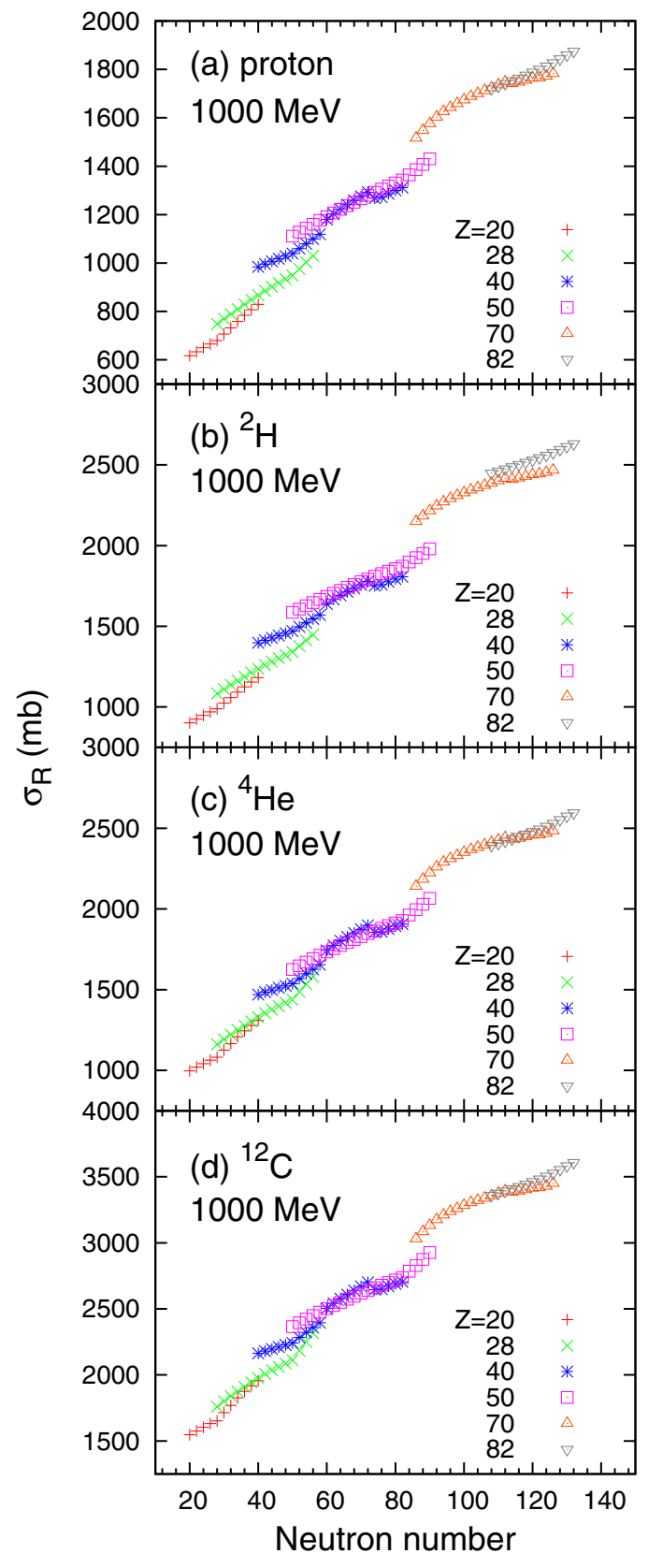

FIG. 8. Total reaction cross sections, $\sigma_{R}^{(N)}+\sigma_{C}$, of various nuclei at $1000 \mathrm{MeV}$ incident energy. The Coulomb breakup contribution is calculated by the EPM. The targets are; (a) proton, (b) ${ }^{2} \mathrm{H}$, (c) ${ }^{4} \mathrm{He}$, and $(\mathrm{d}){ }^{12} \mathrm{C}$. The $\mathrm{SkM}^{*}$ interaction is used.

provides us with a simple formula for including the Coulomb breakup but to determine the cutoff parameter $b_{\max }$ causes some ambiguity. It is important to quantify the extent to which extracting the nuclear radius with $\sigma_{R}$ includes uncertainty due to the Coulomb breakup.

Figure 8 plots the $\sigma_{R}$ 's of isotopes with $Z=20,28,40,50$, 70 , and 82 on ${ }^{1,2} \mathrm{H},{ }^{4} \mathrm{He}$, and ${ }^{12} \mathrm{C}$ targets, as functions of the neutron number. The incident energy is set to be $1000 \mathrm{MeV}$ where the $E 1$ contribution is dominant. The cross section reflects the sizes of both the target and the projectile if the Coulomb breakup contribution is neglected. Since the ${ }^{2} \mathrm{H}$ target 


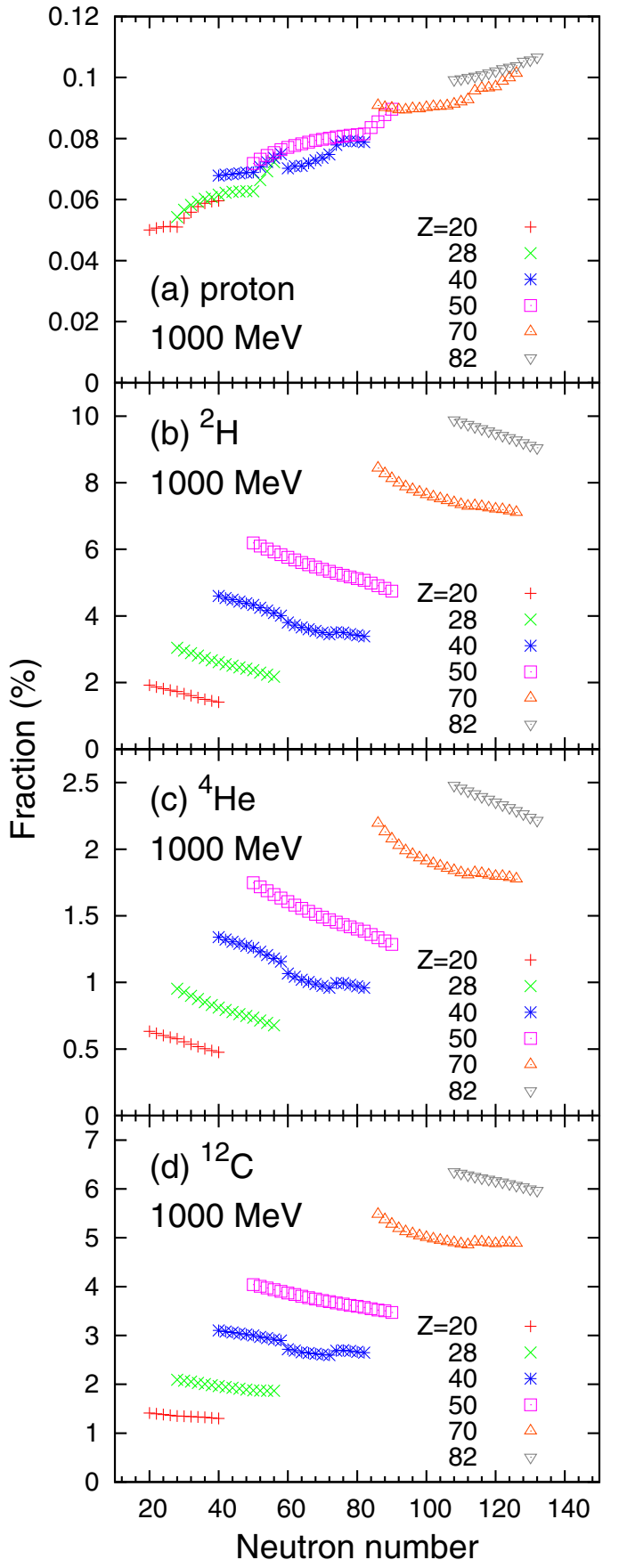

FIG. 9. Same as Fig. 8 but for a fraction of the Coulomb breakup contribution to the total reaction cross section.

has large matter radius and Coulomb breakup cross section, its $\sigma_{R}$ is comparable with that on the ${ }^{4} \mathrm{He}$ target. We display, in Fig. 9, a fraction of the Coulomb breakup cross section to the total reaction cross section, $\sigma_{C} / \sigma_{R}$. When the proton target is used, the contribution is small less than about $0.1 \%$ of $\sigma_{R}$. The proton target can be safely used for extracting the sizes for heavy nuclei even for lead isotopes. The ${ }^{12} \mathrm{C}$ target, which is the most commonly used target, gives at most $6 \%$ of $\sigma_{R}$ for the Coulomb contribution. The ${ }^{4} \mathrm{He}$ target can be used as an alternative to the ${ }^{12} \mathrm{C}$ target, if a preparation for the

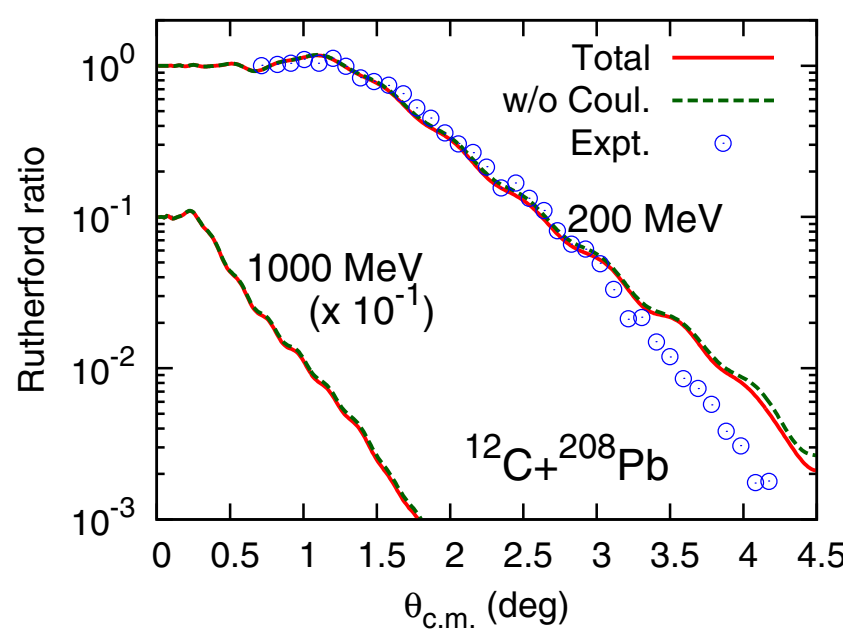

FIG. 10. Rutherford ratios of ${ }^{12} \mathrm{C}+{ }^{208} \mathrm{~Pb}$ elastic scattering at 200 and $1000 \mathrm{MeV}$ incident energies. The ratio calculated by ignoring the Coulomb breakup is also plotted for comparison. The cutoff impact parameter $b_{\max }$ is 15.1 and $80.0 \mathrm{fm}$ for 200 and $1000 \mathrm{MeV}$, respectively. The SkM* interaction is employed. The experimental data are taken from Ref. [69].

target is easier than that for the proton target. The ${ }^{2} \mathrm{H}$ target has smallest charge but gives large Coulomb contribution due to the target dissociation. The contribution is at largest $10 \%$. Since we cannot neglect the Coulomb contribution in the analysis of the rms radius through $\sigma_{R}$, the ${ }^{2} \mathrm{H}$ and ${ }^{12} \mathrm{C}$ targets are disadvantageous in precisely determining the matter radius for heavy projectiles due to unavoidable uncertainty from the Coulomb excitations.

\section{E. Comparison with experimental data}

We have to make sure that the inputs needed in the Glauber model calculation are chosen properly, that is, they should well reproduce available experimental data. To this end, we examine the elastic scattering cross section

$$
\frac{d \sigma}{d \Omega}=|F(\boldsymbol{q})|^{2}
$$

with the elastic scattering amplitude

$$
F(\boldsymbol{q})=F_{C}(q)+i K \int_{0}^{b_{\max }} d b b e^{i \boldsymbol{q} \cdot \boldsymbol{b}+i \chi_{C}(b)}\left(1-e^{i \chi(\boldsymbol{b})}\right),
$$

where $F_{C}(q)$ is the Rutherford phase for the Coulomb elastic scattering and $\chi_{C}(b)=2 Z_{P} Z_{T} \eta \ln (K b)$, and $\boldsymbol{q}$ is a momentum transfer vector. The relativistic kinematics is used for the wave number $K$. Note that the optical-phase shift function $\chi(\boldsymbol{b})$, a key quantity to determine $\sigma_{R}$, appears here as well. The cutoff parameter $b_{\max }$ is determined by the same way as in Sec. III C. Once $b_{\max }$ is determined, the nuclear-Coulomb interference effect is fully taken into account, which is one of the advantages of the Glauber formalism.

Figure 10 plots the Rutherford ratio of ${ }^{208} \mathrm{~Pb}+{ }^{12} \mathrm{C}$ scattering at the incident energy of $200 \mathrm{MeV}$ where experimental data are available [69], and at the incident energy of $1000 \mathrm{MeV}$. The calculation reproduces the data very well up to 3 degrees. 


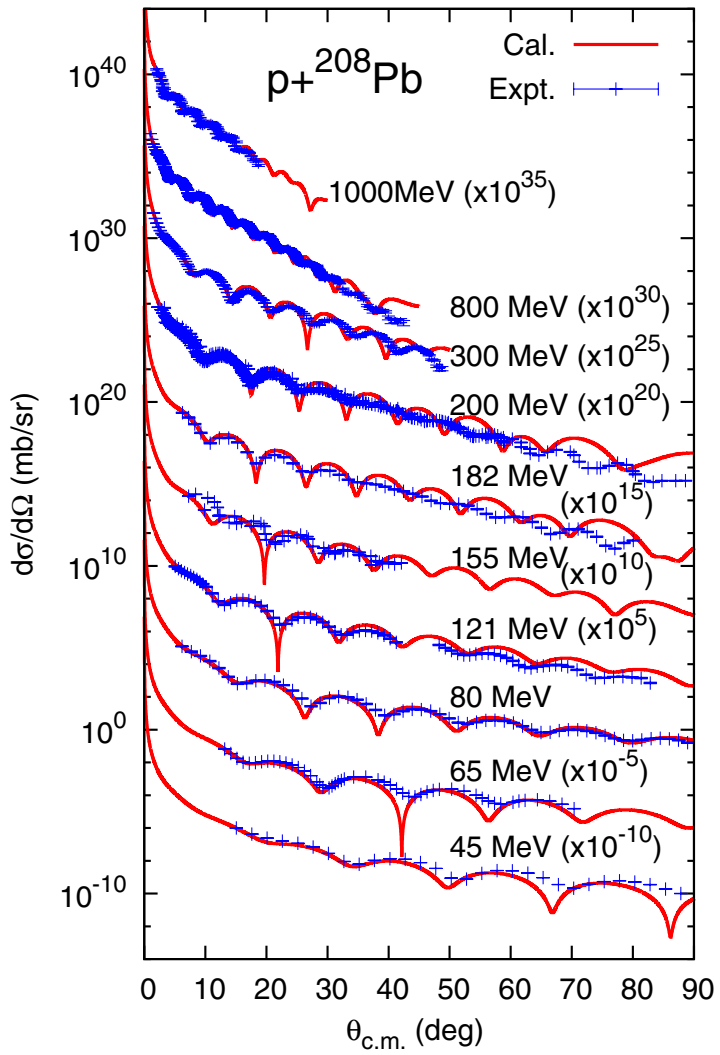

FIG. 11. Elastic differential cross sections of $p+{ }^{208} \mathrm{~Pb}$ scattering. Coulomb breakup contribution is ignored. The SkM* interaction is used. Experimental data are taken from Refs. [13,70-84].

The density distributions and the profile function employed are reliable for describing the forward angle reaction. Because the calculations with and without the Coulomb breakup give only a minor difference, we see virtually no Coulomb effect. Note that at lower energies the condition (28) to determine $b_{\max }$ may not be justified because other multipoles than the dipole contribute. The cut-off parameters $b_{\max }$ for the Glauber model calculation are 15.1 and $80.0 \mathrm{fm}$ for 200 and $1000 \mathrm{MeV}$, respectively. In fact, $b_{\max }$ for $200 \mathrm{MeV}$ appears to be too small, that is, only slightly larger than the touching distance of the ${ }^{12} \mathrm{C}$ and ${ }^{208} \mathrm{~Pb}$, $b_{\min }=10.2 \mathrm{fm}$. The Coulomb breakup estimation is more reliable at $1000 \mathrm{MeV}$ because the $E 1$ transition dominates as already discussed in Fig. 7.

Next we assess the reliability of our model using the protonnucleus collision. Figure 11 plots the differential cross sections of $p+{ }^{208} \mathrm{~Pb}$ scattering in a wide range of the incident energy. We ignore the Coulomb breakup contributions since they are much smaller than those by the ${ }^{12} \mathrm{C}$ target. The calculated cross sections almost perfectly reproduce the experimental data from low to high incident energies. Our calculation has no adjustable parameters but nevertheless well reproduces experiment as equally as the folding-model calculation at 65 to $200 \mathrm{MeV}$ with a recent $G$-matrix interaction [85]. This confirms that our reaction model for the proton-nucleus scattering is reliable for the wide range of the incident energies.

Finally we compare in Fig. 12 the calculated proton-nucleus total reaction cross sections with available experimental data.

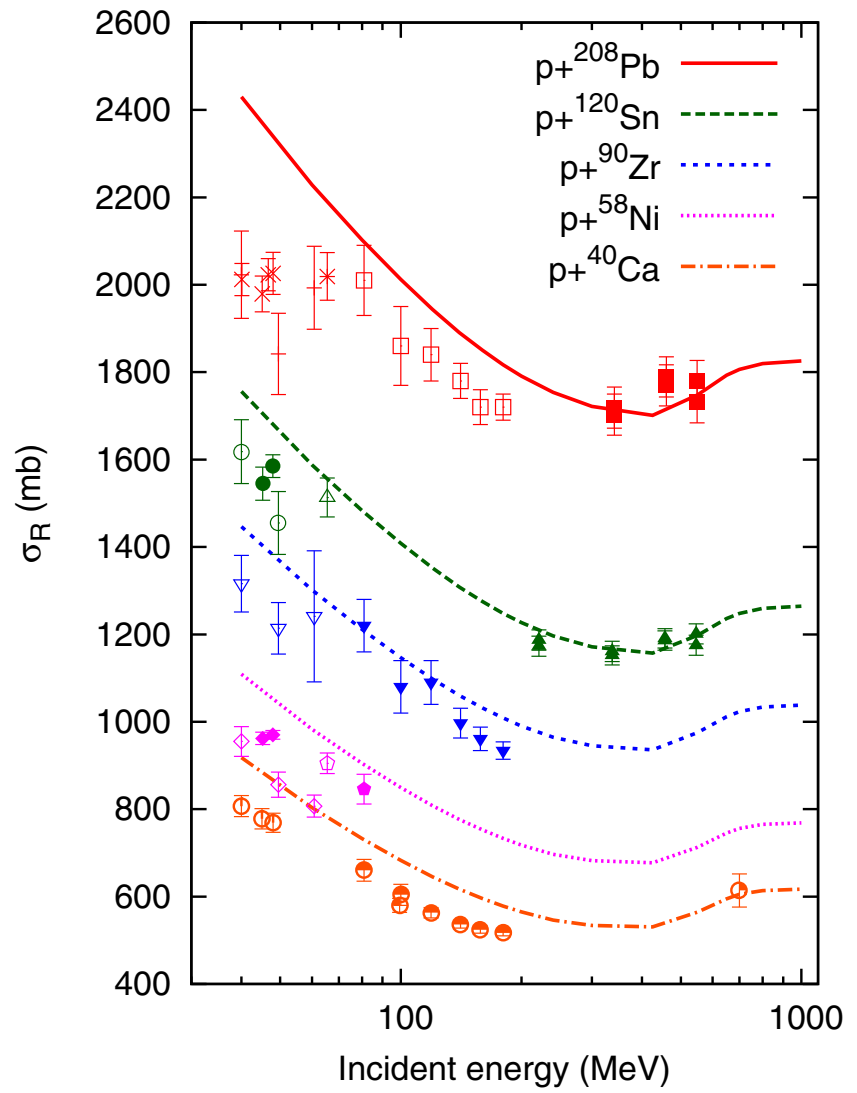

FIG. 12. Total reaction cross sections of ${ }^{40} \mathrm{Ca},{ }^{58} \mathrm{Ni},{ }^{90} \mathrm{Zr},{ }^{120} \mathrm{Sn}$, and ${ }^{208} \mathrm{~Pb}$ on a proton target as functions of incident energies. Coulomb breakup contribution is ignored. The $\mathrm{SkM}^{*}$ interaction is used. The experimental data with error bars are taken from Refs. [86-93].

Since the data before 1970s are very much scattered, we use the experimental data after 1970s. The theory obtains overall agreement with the measured values, especially with the data at the incident energy higher than $200 \mathrm{MeV}$. For ${ }^{90} \mathrm{Zr}$ and

${ }^{120} \mathrm{Sn}$, even at low energy of about $60 \mathrm{MeV}$, the theoretical cross sections are consistent with the measurement within the error bar. For the other systems, the energy dependence of the low-energy cross sections is well reproduced but the theory tends to overestimate the experimental cross sections at around $50 \mathrm{MeV}$. One may think that the applicability of the Glauber model is questionable in such low-energy scattering around $50 \mathrm{MeV}$. In fact in-medium effects such as Pauli blocking and Fermi-motion can be important at low energies and may modify the free $N N$ scattering parameters [94,95]. A fully consistent implementation of such effects in the Glauber theory is, however, not available yet.

\section{F. Correlation between reaction radius and nuclear sizes}

As we have discussed, any targets other than the proton cause some ambiguities concerning the Coulomb breakup contribution that exhibits not only the incident energy dependence but also higher multipole Coulomb excitations. To avoid such uncertainty, the proton target is best to extract the nuclear sizes. As shown in Sec. III E, our model well reproduces 


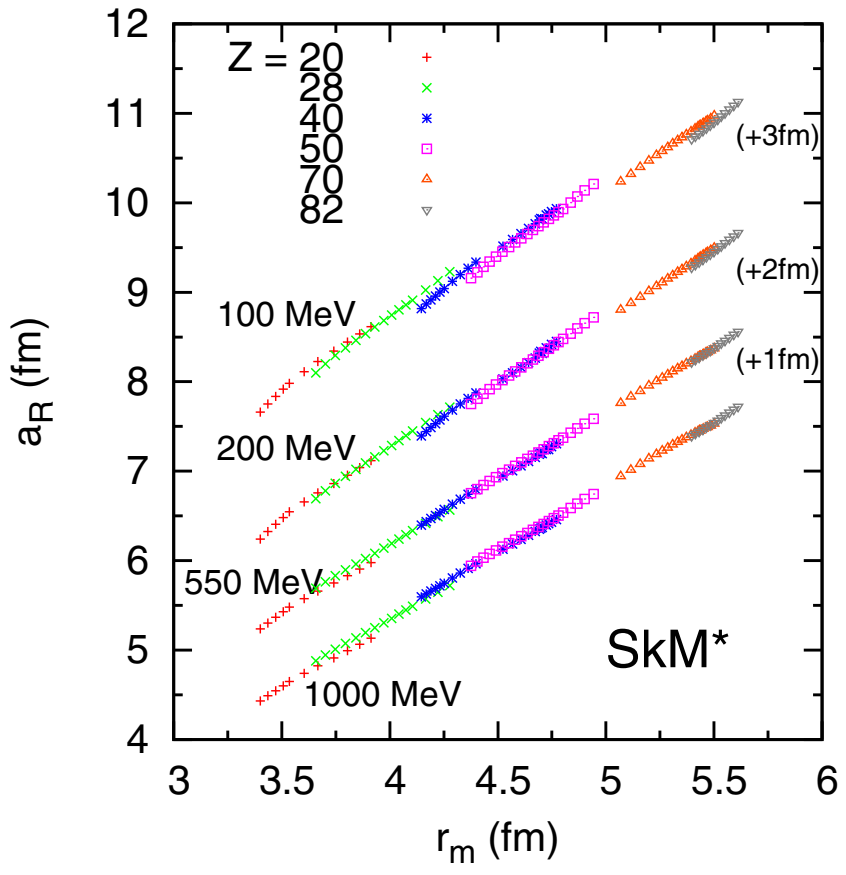

FIG. 13. Reaction radii vs point matter rms radii for $A=40-214$ with $Z=20,28,40,50,70$, and 82 nuclei incident on a proton target at $100,200,550$, and $1000 \mathrm{MeV}$. The reaction radii of 550, 200, and $100 \mathrm{MeV}$ are, respectively, shifted by 1,2 , and $3 \mathrm{fm}$ for clarity. The $\mathrm{SkM}^{*}$ interaction is used.

the experimental cross sections. Therefore, by extending the projectile from medium to heavy nuclei we can reexamine the proposal [26] to make use of the total reaction cross section on the proton target to extract the matter radius and the neutron-skin thickness. For this purpose we carefully analyze the dependence of $\sigma_{R}$ on those quantities.

A 'reaction radius', $a_{R}$, is defined as a measure of the nuclear size that is extracted from the proton-nucleus collision at incident energy $E$ [26]

$$
a_{R}(N, Z, E)=\sqrt{\sigma_{R}(N, Z, E) / \pi} .
$$

The reaction radius may somewhat be related to the one defined in the black sphere model $[96,97]$. The $a_{R}$ depends on $E$ through the different energy-dependence of the $n p$ and $p p$ total cross sections, $\sigma_{n p}^{\text {tot }}$ and $\sigma_{p p}^{\text {tot }}$ [98], that enter the profile function (5). This energy dependence most clearly shows up in $\sigma_{R}$ for the proton target. This is because the ${ }^{2} \mathrm{H},{ }^{4} \mathrm{He}$, and ${ }^{12} \mathrm{C}$ targets contain the equal number of protons and neutrons and their density distributions are almost identical.

To explore the dependence of $a_{R}$ on $E$ and the nuclear sizes, we make use of our $a_{R}$ values obtained by the Glauber model calculation with the HF+BCS densities. Figure 13 displays the correlation diagram of $a_{R}$ vs $r_{m}$ in the case of the proton target. Four incident energies are chosen to show the energy dependence: 100 and $200 \mathrm{MeV}$ where $\sigma_{p p}<\sigma_{n p}$, $550 \mathrm{MeV}$ where $\sigma_{p p} \simeq \sigma_{n p}$, and $1000 \mathrm{MeV}$ where $\sigma_{p p}$ becomes a little larger than $\sigma_{n p}$ [98]. As already noted in Ref. [26], $a_{R}$ approximately follows a straight line but some scattered distributions are observed at the low incident energies. The

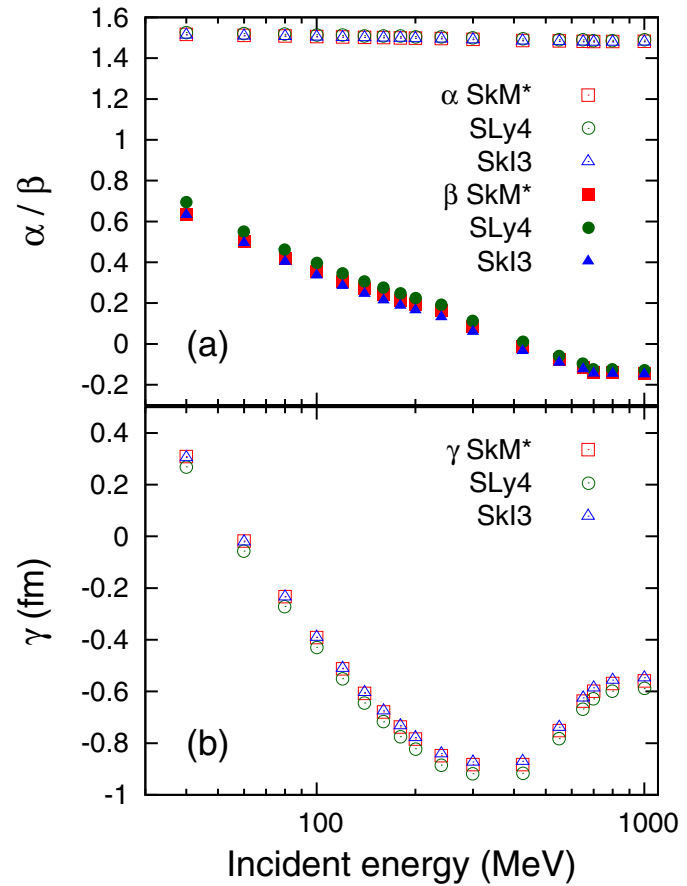

FIG. 14. Incident energy dependence of the coefficients of the empirical formula for the reaction radius (33) calculated with the SkM*, SLy4, and SkI3 interactions: (a) $\alpha(E)$ and $\beta(E)$, and (b) $\gamma(E)$.

latter is understood by considering that at those energies the proton target tends to be more sensitive to the neutron distribution due to its larger $\sigma_{n p}$ than $\sigma_{p p}$.

The sensitivity was disclosed in the behavior of the incidentenergy-dependent coefficients of the empirical formula for $a_{R}[26]$. The $a_{R}$ is very well approximated by a linear function of two variables, $r_{m}$ and $\delta$, as

$a_{R}(N, Z, E) \simeq \alpha(E) r_{m}(N, Z)+\beta(E) \delta(N, Z)+\gamma(E)$,

where $\alpha(E), \beta(E)$, and $\gamma(E)$ are the energy-dependent coefficients that will be determined empirically. Note that the above formula should be more accurate than the one assumed by Carlson [99] that has no $\delta(N, Z)$ term. The energy-dependent coefficients are determined as in the previous procedure. That is, the $a_{R}$ values extracted from $\sigma_{R}$ obtained with the HF+BCS densities are fit in the form (33) by the least-square method. We calculate the mean square deviation

$$
\chi^{2}(E)=\frac{1}{\mathcal{N}} \sum_{N, Z}\left[a_{R}(N, Z, E)-a_{R}^{\mathrm{Fit}}(N, Z, E)\right]^{2},
$$

where $\mathcal{N}$, the number of nuclei considered, is 103 for all the interactions. For all incident energies, the square roots of $\chi^{2}(E)$ are small, resulting in the range of $0.01-0.02 \mathrm{fm}$. Figure 14 plots the coefficients of the empirical formula (33) as a function of the incident energy. Results calculated with the three Skyrme interactions are displayed to show the sensitivity to the different density profiles. For example, when the nucleus shows some deformation, the density profile becomes quite different from that with the spherical one as exemplified in Fig. 1 and in Ref. [21]. In spite of those differences, the three Skyrme interactions lead to virtually the same coefficients. 


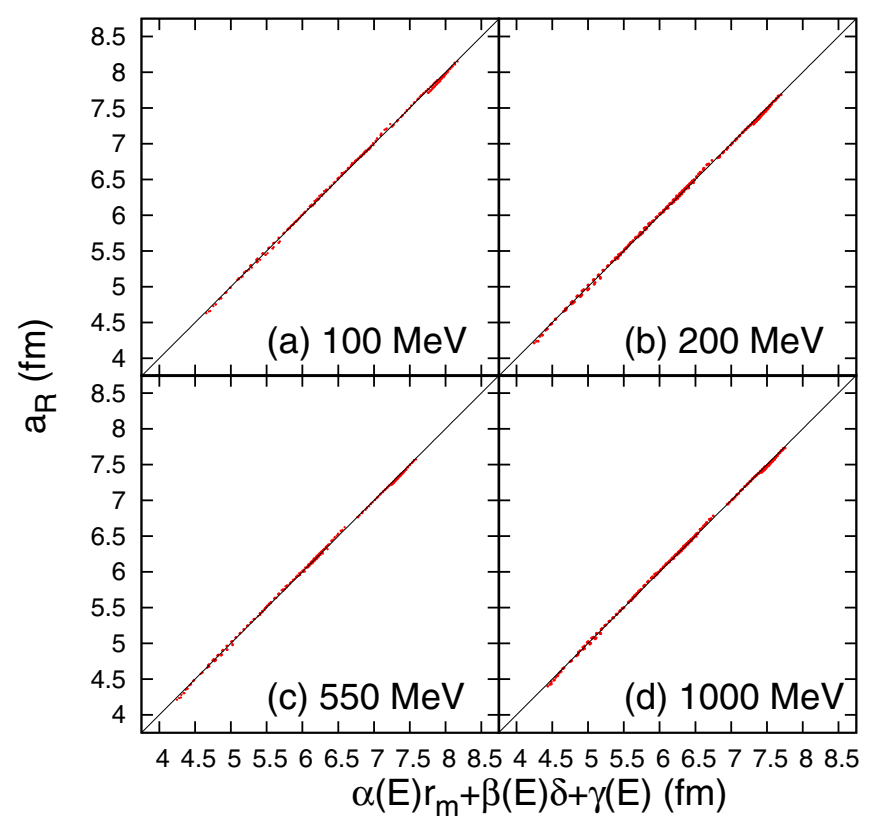

FIG. 15. Correlation diagrams of the reaction radii obtained through the $\mathrm{HF}+\mathrm{BCS}$ densities and by the empirical formula (33) for incident energies of (a) 100, (b) 200, (c) 550, and (d) $1000 \mathrm{MeV}$. All the data points obtained with the $\mathrm{SkM}^{*}$, SLy4, and SkI3 interactions are plotted in dots. A solid line, $y=x$, is drawn as a guide of eyes.

The first term, $\alpha(E) r_{m}$, is the leading term of $a_{R}$, which is a basis to extract the matter radius from $\sigma_{R}$. The coefficient $\beta(E)$ signals the sensitivity of $a_{R}$ to $\delta$. In fact, $\beta(E)$ is positive at the low incident energy, turns to be almost zero at $550 \mathrm{MeV}$ where $\sigma_{n p} \sim \sigma_{p p}$, and becomes negative at higher energies because of $\sigma_{n p}<\sigma_{p p}$. This sensitivity enables us to use the proton target as a probe of the neutron-skin thickness. The last term $\gamma(E)$ follows the energy dependence of the total $N N$ cross sections [98]. This energy-dependence of the coefficients is also found for light to medium nuclei [26] and their values are consistent with those obtained here. The $\alpha(E)$ does not strongly depend on the incident energy, which led to a linear approximation in $\delta$ for the difference of the reaction radii at two incident energies [26]

$$
\Delta a_{R}\left(N, Z, E^{\prime}, E\right)=a_{R}\left(N, Z, E^{\prime}\right)-a_{R}(N, Z, E) .
$$

In the present case the $\Delta a_{R}\left(N, Z, E^{\prime}, E\right)$ vs $\delta$ plot shows some deviation from the linearity because the term, $\left(\alpha\left(E^{\prime}\right)-\right.$ $\alpha(E)) r_{m}(N, Z)$, depending on the matter radius, cannot be completely neglected in the heavy nuclei.

Figure 15 displays the correlation diagram of the reaction radii, $a_{R}$ obtained from $\sigma_{R}$ calculated using the $\mathrm{HF}+\mathrm{BCS}$ density and $a_{R}^{\text {Fit }}$ of the empirical formula (33). All the 309 points follow a straight $y=x$ line very well. We confirm that the coefficients are universal even in heavy nuclei and does not depend on the details of the density profile. The $a_{R}$ is well approximated by Eq. (33), which makes it possible to extract the matter and neutron-skin thickness using the total reaction cross sections on the proton target.
TABLE I. Total reaction cross sections in mb of $p+{ }^{208} \mathrm{~Pb}$ reactions as functions of the neutron-skin thickness $\delta$ ( $\mathrm{fm}$ ) and the incident energy $E(\mathrm{MeV})$. The Fermi-type density distributions are assumed for both protons and neutrons. The proton radius of ${ }^{208} \mathrm{~Pb}$ is set to fit the empirical value, $5.44 \mathrm{fm}$ [100].

\begin{tabular}{lcccccc}
\hline \hline$E$ & $\delta=0.0$ & 0.1 & 0.2 & 0.3 & 0.4 & 0.5 \\
\hline 100 & 1974 & 2014 & 2056 & 2098 & 2142 & 2188 \\
120 & 1904 & 1942 & 1982 & 2023 & 2065 & 2108 \\
140 & 1850 & 1888 & 1926 & 1965 & 2006 & 2047 \\
160 & 1809 & 1846 & 1883 & 1921 & 1960 & 2000 \\
180 & 1777 & 1812 & 1848 & 1885 & 1923 & 1962 \\
200 & 1750 & 1785 & 1820 & 1856 & 1893 & 1932 \\
240 & 1714 & 1747 & 1781 & 1816 & 1851 & 1888 \\
300 & 1684 & 1716 & 1747 & 1780 & 1814 & 1848 \\
425 & 1668 & 1697 & 1727 & 1757 & 1788 & 1820 \\
550 & 1721 & 1749 & 1777 & 1807 & 1838 & 1870 \\
650 & 1771 & 1799 & 1827 & 1856 & 1887 & 1919 \\
700 & 1787 & 1814 & 1841 & 1870 & 1901 & 1932 \\
800 & 1801 & 1828 & 1856 & 1885 & 1916 & 1947 \\
1000 & 1807 & 1834 & 1862 & 1891 & 1921 & 1953 \\
\hline \hline
\end{tabular}

\section{G. Feasibility of measurements to determine neutron-skin thickness of ${ }^{208} \mathrm{~Pb}$}

We have shown that $a_{R}$ or $\sigma_{R}$ on the proton target is model independent, that is, only depends on the matter radius and neutron-skin thickness. Thus far, several experimental studies have been performed to determine the neutron-skin thickness of ${ }^{208} \mathrm{~Pb}$. The model independent analysis by the parityviolating elastic electron scattering [8] gives the neutron-skin thickness within an accuracy of $0.2 \mathrm{fm}$. Here we examine how much accuracy of $\sigma_{R}$ measurement on the proton target is needed to deduce the skin thickness of ${ }^{208} \mathrm{~Pb}$ more reliably.

First, we assume Fermi-type density distributions for both protons and neutrons with a common diffuseness parameter, $a$, and the proton radius parameter $R_{p}$ is set to reproduce the measured proton radius of ${ }^{208} \mathrm{~Pb}(5.44 \mathrm{fm}$ [100]). The neutron radius parameter $R_{n}$ controls the neutron radius or $\delta$. Table I lists the $\sigma_{R}$ values calculated with different $\delta$ parameters as a function of the incident energy. The Coulomb breakup contribution is neglected because it is typically $1 \mathrm{mb}$ and its energy dependence is also very small, see Fig. 4. At the lowest incident energy of $100 \mathrm{MeV}$, where the sensitivity to the skin thickness becomes largest, the increase of $\sigma_{R}$ is large, approximately $50 \mathrm{mb}$ for every increase of $\delta$ by $0.1 \mathrm{fm}$. If the neutron-skin thickness should be determined within $0.1 \mathrm{fm}$ accuracy, the required accuracy of the measurement has to be a few percent. With the increasing incident energy, the change of the cross section becomes smaller. A measurement with lower incident energy is therefore more advantageous to extract the skin thickness. Of course it must be high enough to assure the validity of the Glauber model.

If the proton radius is not known, we need to measure at least two cross sections at low and high incident energies, and then determine $r_{p}$ and $r_{n}$ so as to reproduce those cross sections simultaneously. In order to maximize the sensitivity of $\sigma_{R}$ to $\delta$, it is better to choose the low energy where $\beta(E)$ is large and the high energy where $\beta(E)$ is small. Table II lists the 
TABLE II. Neutron-skin thickness dependence of the difference of $p+{ }^{208} \mathrm{~Pb}$ total reaction cross sections at two incident energies, $\sigma_{R}(E)-\sigma_{R}\left(E^{\prime}\right)$. Units of cross section, energy, and length are mb, $\mathrm{MeV}$, and fm, respectively.

\begin{tabular}{lrrrrrr}
\hline \hline$\left(E, E^{\prime}\right)$ & $\delta=0.0$ & 0.1 & 0.2 & 0.3 & 0.4 & 0.5 \\
\hline$(100,550)$ & 253 & 265 & 278 & 291 & 304 & 318 \\
$(100,1000)$ & 166 & 180 & 194 & 207 & 220 & 235 \\
$(200,550)$ & 29 & 36 & 43 & 49 & 56 & 62 \\
$(200,1000)$ & -56 & -49 & -42 & -35 & -28 & -21 \\
\hline \hline
\end{tabular}

difference of $\sigma_{R}$ 's calculated at different incident energies. As seen in Table II, the cross section difference becomes largest for a choice of the incident energies, 100 and $550 \mathrm{MeV}$, and it is in the range of $250-320 \mathrm{mb}$, depending on the value of $\delta$. This value is more than $10 \%$ of $\sigma_{R}$ itself, and therefore can easily be measured with the accuracy of the present-day cross section measurements. To determine the neutron-skin thickness, a finer resolution is needed to distinguish a change of $\delta$. As shown in Table II, the cross section difference is at most $20 \mathrm{mb}$ for every change of $\delta$ by $0.1 \mathrm{fm}$. To detect this change, one has to measure $\sigma_{R}$ at least with an accuracy of $1 \%$, and then it is possible to determine the skin thickness within the uncertainty of $0.1 \mathrm{fm}$. If one chooses 200 and $550 \mathrm{MeV}$, the cross section difference becomes much less sensitive to the change of $\delta$, and $\sigma_{R}$ has to be measured at least within the accuracy of $0.5 \%$ to determine the neutron-skin thickness within the uncertainty of $0.1 \mathrm{fm}$ accuracy.

To reduce the accuracy needed for the size extraction, we recommend that $\sigma_{R}$ is measured at $100 \mathrm{MeV}$ and at $550 \mathrm{MeV}$ or higher energy. To achieve this, $\sigma_{R}$ measurement on the proton target has to be improved up to the level of the ${ }^{12} \mathrm{C}$ target, which has been performed in $1 \%$ [11].

\section{CONCLUSIONS}

To facilitate the study of nuclear size properties of heavy nuclei, we have made a systematic analysis of total reaction cross sections, $\sigma_{R}$, on various light targets, ${ }^{1,2} \mathrm{H},{ }^{4} \mathrm{He}$, and ${ }^{12} \mathrm{C}$ with the Glauber model. In order to make the systematic evaluation of the total reaction cross sections for a wide range of mass numbers, the Skyrme-Hartree-Fock+BCS model is applied to generate the density distributions for more than hundred even-even nuclei with $A=40-214$ selected from among $\mathrm{Ca}, \mathrm{Ni}, \mathrm{Zr}, \mathrm{Sn}, \mathrm{Yb}$, and $\mathrm{Pb}$ isotopes. Three different interactions, $\mathrm{SkM}^{*}$, SLy4, and SkI3 are employed. The Coulomb breakup contributions to $\sigma_{R}$ are systematically investigated by the equivalent-photon method (EPM) using the electric dipole $(E 1)$ strength function obtained by the time-dependent mean-field theory, that is, the canonical-basistime-dependent-Hartree-Fock-Bogoliubov theory.

We have analyzed the Coulomb breakup contribution to the total reaction cross section based on the optical limit approximation of the Glauber theory. The divergence problem of the eikonal Coulomb phase is avoided by introducing the cutoff impact parameter determined so as to reproduce the Coulomb breakup cross section obtained by the EPM. We have compared the reaction probabilities obtained by the Glauber and EPM calculations and found out that the EPM with the $E 1$ approximation gives a fair description of the Coulomb breakup processes for high energy heavy ion collisions.

Our analysis gives an estimate for the Coulomb breakup contribution that is useful for a future study of heavy nuclei. The Coulomb contributions are not negligible for large- $Z$ nuclei using the ${ }^{12} \mathrm{C}$ target, which is one of the targets commonly used for extracting the matter radius of light nuclei. A target with smaller $Z$, e.g., ${ }^{4} \mathrm{He}$, gives smaller Coulomb breakup cross sections. However, the use of ${ }^{2} \mathrm{H}$ target gives even worse results because the contribution of the Coulomb excitation is extremely large. The analysis shows the difficulty to find out a general trend of the Coulomb breakup cross sections. They depend on the target, incident energy, and interactions employed for the projectile. Higher multipole effects other than the $E 1$ contribution need to be taken into account for a quantitative analysis at incident energies lower than $550 \mathrm{MeV}$.

A proton target is the best target to extract the nuclear sizes because the complexities of the Coulomb breakup process can be avoided. We show that the reaction radius, $a_{R}=\sqrt{\sigma_{R} / \pi}$, on the proton target actually probes the sizes of a nucleus and has sensitivity to the neutron-skin thickness. In fact the reaction radius is very well approximated by a linear function of $r_{m}$ and $\delta$. The incident-energy-dependent coefficients are determined by the least-square fitting using the density distributions of medium to heavy nuclei obtained by the HF+BCS. It is found that the coefficients are universal functions that depend only on the incident energy and do not depend on the interaction employed or density profiles.

A required accuracy to extract the neutron-skin thickness has been examined in the case of $p+{ }^{208} \mathrm{~Pb}$. Assuming that the proton radius of ${ }^{208} \mathrm{~Pb}$ is known, we need to measure the total reaction cross section within a few percent accuracy in order to determine the neutron-skin thickness and matter radius within the accuracy of $0.1 \mathrm{fm}$. However, if the proton radius of the projectile nucleus is unknown, less than $1 \%$ accuracy is required to determine the skin thickness at the accuracy of $0.1 \mathrm{fm}$. Practically, we have to measure the total reaction cross sections on the proton target at both low and high incident energies, and to extract the neutron and proton radii consistently with the measured cross sections. Here 'low' means typically 100-200 MeV where the difference of $\sigma_{n p}$ and $\sigma_{p p}$ is considerably large and in addition the Glauber approximation can be safely applied. If we could know the matter (proton) radius by any methods, one needs to measure only one total reaction cross section at the low (any) energy where the cross section has more sensitivity to the neutron distribution. In that case, the required accuracy for the cross section measurement will be reduced.

We conclude that making use of the energy dependence of the total reaction cross sections on the proton target is a most promising way to extract the matter and neutron-skin thickness for medium to heavy unstable nuclei.

\section{ACKNOWLEDGMENTS}

The authors are grateful to B. V. Carlson for his careful reading of the manuscript. They also thank $\mathrm{T}$. Inakura 
for supplying his code to calculate the angle-averaged one-body density. The work was in part supported by
JSPS KAKENHI Grant Nos. (24540261, 25800121, and 15K05072).
[1] P. B. Demorest, T. Pennucci, S. M. Ransom, M. S. E. Roberts, and J. W. T. Hessels, Nature 467, 1081 (2010).

[2] L. W. Chen, C. M. Ko, B. A. Li, and J. Xu, Phys. Rev. C 82, 024321 (2010).

[3] P.-G. Reinhard and W. Nazarewicz, Phys. Rev. C 81, 051303(R) (2010).

[4] X. Roca-Maza, M. Centelles, X. Viñas, and M. Warda, Phys. Rev. Lett. 106, 252501 (2011).

[5] M. Kortelainen, J. Erler, W. Nazarewicz, N. Birge, Y. Gao, and E. Olsen, Phys. Rev. C 88, 031305(R) (2013).

[6] T. Inakura, T. Nakatsukasa, and K. Yabana, Phys. Rev. C 88, 051305(R) (2013).

[7] X. Roca-Maza, X. Viñas, M. Centelles, B. K. Agrawal, G. Colò, N. Paar, J. Piekarewicz, and D. Vretenar, Phys. Rev. C 92, 064304 (2015).

[8] S. Abrahamyan et al., Phys. Rev. Lett. 108, 112502 (2012).

[9] R. Kanungo, A. Prochazka, W. Horiuchi, C. Nociforo, T. Aumann, D. Boutin, D. Cortina-Gil, B. Davids, M. Diakaki, F. Farinon, H. Geissel, R. Gernhauser, J. Gerl, R. Janik, B. Jonson, B. Kindler, R. Knobel, R. Krucken, M. Lantz, H. Lenske, Y. Litvinov, B. Lommel, K. Mahata, P. Maierbeck, A. Musumarra, T. Nilsson, C. Perro, C. Scheidenberger, B. Sitar, P. Strmen, B. Sun, Y. Suzuki, I. Szarka, I. Tanihata, Y. Utsuno, H. Weick, and M. Winkler, Phys. Rev. C 83, 021302(R) (2011).

[10] M. Takechi et al., Mod. Phys. Lett. A 25, 1878 (2010).

[11] M. Takechi et al., Phys. Rev. C 90, 061305(R) (2014).

[12] S. Terashima, H. Sakaguchi, H. Takeda, T. Ishikawa, M. Itoh, T. Kawabata, T. Murakami, M. Uchida, Y. Yasuda, M. Yosoi, J. Zenihiro, H. P. Yoshida, T. Noro, T. Ishida, S. Asaji, and T. Yonemura, Phys. Rev. C 77, 024317 (2008).

[13] J. Zenihiro, H. Sakaguchi, T. Murakami, M. Yosoi, Y. Yasuda, S. Terashima, Y. Iwao, H. Takeda, M. Itoh, H. P. Yoshida, and M. Uchida, Phys. Rev. C 82, 044611 (2010).

[14] A. Tamii, I. Poltoratska, P. von Neumann-Cosel, Y. Fujita, T. Adachi, C. A. Bertulani, J. Carter, M. Dozono, H. Fujita, K. Fujita, K. Hatanaka, D. Ishikawa, M. Itoh, T. Kawabata, Y. Kalmykov, A. M. Krumbholz, E. Litvinova, H. Matsubara, K. Nakanishi, R. Neveling, H. Okamura, H. J. Ong, B. Ozel-Tashenov, V. Y. Ponomarev, A. Richter, B. Rubio, H. Sakaguchi, Y. Sakemi, Y. Sasamoto, Y. Shimbara, Y. Shimizu, F. D. Smit, T. Suzuki, Y. Tameshige, J. Wambach, R. Yamada, M. Yosoi, and J. Zenihiro, Phys. Rev. Lett. 107, 062502 (2011).

[15] D. T. Yordanov, M. L. Bissell, K. Blaum, M. De Rydt, C. Geppert, M. Kowalska, J. Krämer, K. Kreim, A. Krieger, P. Lievens, T. Neff, R. Neugart, G. Neyens, W. Nörtershäuser, R. Sánchez, and P. Vingerhoets, Phys. Rev. Lett. 108, 042504 (2012).

[16] W. Géithner, T. Neff, G. Audi, K. Blaum, P. Delahaye, H. Feldmeier, S. Géorge, C. Guenaut, F. Herfurth, A. Herlert, S. Kappertz, M. Keim, A. Kellerbauer, H.-J. Kluge, M. Kowalska, P. Lievens, D. Lunney, K. Marinova, R. Neugart, L. Schweikhard, S. Wilbert, and C. Yazidjian, Phys. Rev. Lett. 101, 252502 (2008).

[17] A. Krieger, K. Blaum, M. L. Bissell, N. Frömmgen, C. Geppert, M. Hammen, K. Kreim, M. Kowalska, J. Kramer, T. Neff, R. Neugart, G. Neyens, W. Nörtershäuser, C. Novotny,
R. Sánchez, and D. T. Yordanov, Phys. Rev. Lett. 108, 142501 (2012).

[18] T. Sumi, K. Minomo, S. Tagami, M. Kimura, T. Matsumoto, K. Ogata, Y. R. Shimizu, and M. Yahiro, Phys. Rev. C 85, 064613 (2012).

[19] S. Watanabe, K. Minomo, M. Shimada, S. Tagami, M. Kimura, M. Takechi, M. Fukuda, D. Nishimura, T. Suzuki, T. Matsumoto, Y. R. Shimizu, and M. Yahiro, Phys. Rev. C 89, 044610 (2014).

[20] K. Minomo, T. Sumi, M. Kimura, K. Ogata, Y. R. Shimizu, and M. Yahiro, Phys. Rev. C 84, 034602 (2011).

[21] W. Horiuchi, T. Inakura, T. Nakatsukasa, and Y. Suzuki, Phys. Rev. C 86, 024614 (2012).

[22] K. Minomo, T. Sumi, M. Kimura, K. Ogata, Y. R. Shimizu, and M. Yahiro, Phys. Rev. Lett. 108, 052503 (2012).

[23] T. Yamaguchi, I. Hachiuma, A. Kitagawa, K. Namihira, S. Sato, T. Suzuki, I. Tanihata, and M. Fukuda, Phys. Rev. Lett. 107, 032502 (2011).

[24] A. Estradé R. Kanungo, W. Horiuchi, F. Ameil, J. Atkinson, Y. Ayyad, D. Cortina-Gil, I. Dillmann, A. Evdokimov, F. Farinon, H. Geissel, G. Guastalla, R. Janik, M. Kimura, R. Knobel, J. Kurcewicz, Y. A. Litvinov, M. Marta, M. Mostazo, I. Mukha, C. Nociforo, H. J. Ong, S. Pietri, A. Prochazka, C. Scheidenberger, B. Sitar, P. Strmen, Y. Suzuki, M. Takechi, J. Tanaka, I. Tanihata, S. Terashima, J. Vargas, H. Weick, and J. S. Winfield, Phys. Rev. Lett. 113, 132501 (2014).

[25] S. Terashima et al., Prog. Theor. Exp. Phys. (2014) $101 \mathrm{D} 02$.

[26] W. Horiuchi, Y. Suzuki, and T. Inakura, Phys. Rev. C 89, 011601(R) (2014).

[27] P. Ring and P. Schuck, The Nuclear Many-Body Problems, Texts and Monographs in Physics (Springer-Verlag, New York, 1980).

[28] R. J. Glauber, in Lectures in Theoretical Physics, edited by W. E. Brittin and L. G. Dunham (Interscience, New York, 1959), Vol. 1, p. 315.

[29] C. A. Bertulani and G. Baur, Phys. Rep. 163, 299 (1988).

[30] Y. Suzuki, R. G. Lovas, K. Yabana, and K. Varga, Structure and Reactions of Light Exotic Nuclei (Taylor \& Francis, London, 2003).

[31] C. A. Bertulani and P. Danielewicz, Introduction to Nuclear Reactions, Graduate Student Series in Physics (CRC-Taylor \& Francis, London, 2004).

[32] S. Ebata, T. Nakatsukasa, T. Inakura, K. Yoshida, Y. Hashimoto, and K. Yabana, Phys. Rev. C 82, 034306 (2010).

[33] L. Ray, Phys. Rev. C 20, 1857 (1979).

[34] B. Abu-Ibrahim, W. Horiuchi, A. Kohama, and Y. Suzuki, Phys. Rev. C 77, 034607 (2008).

[35] K. Varga, S. C. Pieper, Y. Suzuki, and R. B. Wiringa, Phys. Rev. C 66, 034611 (2002).

[36] B. Abu-Ibrahim and Y. Suzuki, Phys. Rev. C 62, 034608 (2000).

[37] W. Horiuchi, Y. Suzuki, B. Abu-Ibrahim, and A. Kohama, Phys. Rev. C 75, 044607 (2007).

[38] W. Horiuchi and Y. Suzuki, Few-Body Syst. 54, 2407 (2013).

[39] See Supplemental Material at http://link.aps.org/supplemental/ 10.1103/PhysRevC.93.044611 for (1) the parameters of the 
target density distributions and (2) the nuclear properties and the total reaction cross sections on a proton target that are calculated, using the three kinds of Skyrme interactions, for 103 nuclei selected from among $\mathrm{Ca}, \mathrm{Ni}, \mathrm{Zr}, \mathrm{Sn}, \mathrm{Yb}$, and $\mathrm{Pb}$ isotopes.

[40] B. Abu-Ibrahim and Y. Suzuki, Phys. Rev. C 61, 051601(R) (2000).

[41] T. Kido, K. Yabana, and Y. Suzuki, Phys. Rev. C 50, R1276 (1994).

[42] H. Esbensen, G. F. Bertsch, and C. A. Bertulani, Nucl. Phys. A 581, 107 (1995).

[43] D. Baye, P. Capel, and G. Goldstein, Phys. Rev. Lett. 95, 082502 (2005).

[44] G. Goldstein, D. Baye, and P. Capel, Phys. Rev. C 73, 024602 (2006).

[45] M. Yahiro, K. Ogata, and K. Minomo, Prog. Theor. Phys. 126, 167 (2011).

[46] J. Margeron, A. Bonaccorso, and D. M. Brink, Nucl. Phys. A 720, 337 (2003).

[47] B. Abu-Ibrahim and Y. Suzuki, Prog. Theor. Phys. 112, 1013 (2004); 114, 901 (2005).

[48] P. Capel, D. Baye, and Y. Suzuki, Phys. Rev. C 78, 054602 (2008).

[49] D. Baye, P. Capel, P. Descouvemont, and Y. Suzuki, Phys. Rev. C 79, 024607 (2009).

[50] E. C. Pinilla, P. Descouvemont, and D. Baye, Phys. Rev. C 85, 054610 (2012).

[51] W. Horiuchi, Y. Suzuki, P. Capel, and D. Baye, Phys. Rev. C 81, 024606 (2010).

[52] T. Nakamura, A. M. Vinodkumar, T. Sugimoto, N. Aoi, H. Baba, D. Bazin, N. Fukuda, T. Gomi, H. Hasegawa, N. Imai, M. Ishihara, T. Kobayashi, Y. Kondo, T. Kubo, M. Miura, T. Motobayashi, H. Otsu, A. Saito, H. Sakurai, S. Shimoura, K. Watanabe, Y. X. Watanabe, T. Yakushiji, Y. Yanagisawa, and K. Yoneda, Phys. Rev. Lett. 96, 252502 (2006).

[53] T. Nakamura, N. Kobayashi, Y. Kondo, Y. Satou, N. Aoi, H. Baba, S. Deguchi, N. Fukuda, J. Gibelin, N. Inabe, M. Ishihara, D. Kameda, Y. Kawada, T. Kubo, K. Kusaka, A. Mengoni, T. Motobayashi, T. Ohnishi, M. Ohtake, N. A. Orr, H. Otsu, T. Otsuka, A. Saito, H. Sakurai, S. Shimoura, T. Sumikama, H. Takeda, E. Takeshita, M. Takechi, S. Takeuchi, K. Tanaka, K. N. Tanaka, N. Tanaka, Y. Togano, Y. Utsuno, K. Yoneda, A. Yoshida, and K. Yoshida, Phys. Rev. Lett. 103, 262501 (2009).

[54] C. A. Bertulani and A. M. Nathan, Nucl. Phys. A 554, 158 (1993).

[55] K. Yoshida, T. Fukui, K. Minomo, and K. Ogata, Prog. Theor. Exp. Phys. (2014) 053D03.

[56] A. Bohr and B. R. Mottelson, Nuclear Structure, Vols. I and II (W. A. Benjamin, New York, 1975).

[57] J. Terasaki and J. Engel, Phys. Rev. C 82, 034326 (2010).

[58] S. Ebata, T. Nakatsukasa, T. Inakura, K. Yoshida, Y. Hashimoto, and K. Yabana, RIKEN Accel. Prog. Rep. 44, iv (2010).

[59] S. Ebata, T. Nakatsukasa, and T. Inakura, Phys. Rev. C 90, 024303 (2014).

[60] J. Bartel, P. Quentin, M. Brack, C. Guet, and H. Håkansson, Nucl. Phys. A 386, 79 (1982).

[61] E. Chanbanat, P. Bonche, P. Haensel, J. Mayer, and R. Schaeffer, Nucl. Phys. A 627, 710 (1997).

[62] P.-G. Reinhard and H. Flocard, Nucl. Phys. A 584, 467 (1995).

[63] T. Nakatsukasa and K. Yabana, Phys. Rev. C 71, 024301 (2005).
[64] J. Dobaczewski, W. Nazarewicz, T. R. Werner, J. F. Berger, C. R. Chinn, and J. Decharge, Phys. Rev. C 53, 2809 (1996).

[65] N. Tajima, S. Takahara, and N. Onishi, Nucl. Phys. A 603, 23 (1996).

[66] K. Y. Hara, H. Utsunomiya, S. Goko, H. Akimune, T. Yamagata, M. Ohta, H. Toyokawa, K. Kudo, A. Uritani, Y. Shibata, Y. W. Lui, and H. Ohgaki, Phys. Rev. D 68, 072001 (2003).

[67] K. Yabana, Y. Ogawa, and Y. Suzuki, Nucl. Phys. A 539, 295 (1992)

[68] Y. Ogawa, K. Yabana, and Y. Suzuki, Nucl. Phys. A 543, 722 (1992).

[69] J. Y. Hostachy et al., Nucl. Phys. A 490, 441 (1988).

[70] W. T. H. Van Oers et al., Phys. Rev. C 10, 307 (1974).

[71] H. Sakaguchi et al., Phys. Lett. B 99, 92 (1981).

[72] A. Nadasen, P. Schwandt, P. P. Singh, W. W. Jacobs, A. D. Bacher, P. T. Debevec, M. D. Kaitchuck, and J. T. Meek, Phys. Rev. C 23, 1023 (1981).

[73] A. Willis et al., J. Phys. (Paris) 30, 13 (1969).

[74] V. Comparat et al., Nucl. Phys. A 221, 403 (1974).

[75] D. A. Hutcheon et al., Nucl. Phys. A 483, 429 (1988).

[76] L. Lee et al., Phys. Lett. B 205, 219 (1988).

[77] D. K. McDaniels, J. R. Tinsley, J. Lisantti, D. M. Drake, I. Bergqvist, L. W. Swenson, F. E. Bertrand, E. E. Gross, D. J. Horen, T. P. Sjoreen, R. Liljestrand, and H. Wilson, Phys. Rev. C 33, 1943 (1986).

[78] C. Djalali et al., Nucl. Phys. A 380, 42 (1982).

[79] G. W. Hoffmann, L. Ray, M. Barlett, J. McGill, G. S. Adams, G. J. Igo, F. Irom, A. T. M. Wang, C. A. Whitten, R. L. Boudrie, J. F. Amann, C. Glashausser, N. M. Hintz, G. S. Kyle, and G. S. Blanpied, Phys. Rev. C 21, 1488 (1980).

[80] G. S. Blanpied, W. R. Coker, R. P. Liljestrand, G. W. Hoffmann, L. Ray, D. Madland, C. L. Morris, J. C. Pratt, J. E. Spencer, H. A. Thiessen, T. Kozlowski, N. M. Hintz, G. S. Kyle, M. A. Oothoudt, T. S. Bauer, G. Igo, R. J. Ridge, C. A. Whitten, Jr., P. M. Lang, H. Nann, and K. K. Seth, Phys. Rev. C 18, 1436 (1978).

[81] M. M. Gazzaly, N. M. Hintz, G. S. Kyle, R. K. Owen, G. W. Hoffmann, M. Barlett, and G. Blanpied, Phys. Rev. C 25, 408 (1982).

[82] G. S. Blanpied, W. R. Coker, R. P. Liljestrand, L. Ray, G. W. Hoffmann, D. Madland, C. L. Morris, J. C. Pratt, J. E. Spencer, H. A. Thiessen, N. M. Hintz, G. S. Kyle, M. A. Oothoudt, T. S. Bauer, J. C. Fong, G. Igo, R. J. Ridge, C. A. Whitten, T. Kozlowski, D. K. McDaniels, P. Varghese, P. M. Lang, H. Nann, K. K. Seth, and C. Glashausser, Phys. Rev. Lett. 39, 1447 (1977).

[83] G. D. Alkhazov et al., Yad. Fiz. 26, 673 (1977).

[84] R. Bertini et al., Phys. Lett. B 45, 119 (1973).

[85] T. Furumoto, Y. Sakuragi, and Y. Yamamoto, Phys. Rev. C 78, 044610 (2008).

[86] R. F. Carlson et al., Phys. Rev. C 12, 1167 (1975).

[87] B. D. Anderson, P. R. Bevington, F. H. Cverna, M. W. McNaughton, H. B. Willard, R. J. Barrett, N. S. P. King, and D. J. Ernst, Phys. Rev. C 19, 905 (1979).

[88] A. Ingemarsson et al., Nucl. Phys. A 653, 341 (1999).

[89] A. Auce, A. Ingemarsson, R. Johansson, M. Lantz, G. Tibell, R. F. Carlson, M. J. Shachno, A. A. Cowley, G. C. Hillhouse, N. M. Jacobs, J. A. Stander, J. J. van Zyl, S. V. Fortsch, J. J. Lawrie, F. D. Smit, and G. F. Steyn, Phys. Rev. C 71, 064606 (2005). 
[90] J. J. H. Menet et al., Phys. Rev. C 4, 1114 (1971).

[91] T. Eliyakut-Roshko, R. H. McCamis, W. T. H. van Oers, R. F. Carlson, and A. J. Cox, Phys. Rev. C 51, 1295 (1995).

[92] R. F. Carlson et al., Can. J. Phys. 73, 512 (1995).

[93] P. U. Renberg et al., Nucl. Phys. A 183, 81 (1972).

[94] M. S. Hussein, R. A. Rego, and C. A. Bertulani, Phys. Rep. 201, 279 (1991).

[95] M. Takechi, M. Fukuda, M. Mihara, K. Tanaka, T. Chinda, T. Matsumasa, M. Nishimoto, R. Matsumiya, Y. Nakashima, H. Matsubara, K. Matsuta, T. Minamisono, T. Ohtsubo, T. Izumikawa, S. Momota, T. Suzuki, T. Yamaguchi, R. Koyama, W. Shinozaki, M. Takahashi, A. Takizawa, T. Matsuyama,
S. Nakajima, K. Kobayashi, M. Hosoi, T. Suda, M. Sasaki, S. Sato, M. Kanazawa, and A. Kitagawa, Phys. Rev. C 79, 061601(R) (2009).

[96] A. Kohama, K. Iida, and K. Oyamatsu, Phys. Rev. C 69, 064316 (2004).

[97] A. Kohama, K. Iida, and K. Oyamatsu, Phys. Rev. C 72, 024602 (2005).

[98] J. Belinger et al. (Particle Data Group), Phys. Rev. D 86, 010001 (2012).

[99] R. F. Carlson, At. Data Nucl. Data Tables 63, 93 (1996).

[100] I. Angeli and K. P. Marinova, At. Data Nucl. Data Tables 99, 69 (2013). 